\title{
Oncogenic ZEB2/miR-637/HMGA1 signaling axis targeting vimentin promotes the malignant phenotype of glioma
}

Yu Zeng

Shanghai Tenth People's Hospital

Tianshi Que

Southern Medical University Nanfang Hospital

Jie Lin

Southern Medical University Nanfang Hospital

Anqi Xu

Southern Medical University Nanfang Hospital

\section{Zhiyong Wu}

Southern Medical University Nanfang Hospital

Cheng Xie

Southern Medical University Nanfang Hospital

Jie Luo

Southern Medical University Nanfang Hospital

\section{Shengfeng Ding}

Southern Medical University Nanfang Hospital

\section{Zhengming Zhan}

Southern Medical University Nanfang Hospital

Hao Long

Southern Medical University Nanfang Hospital

\section{Xian Zhang}

Southern Medical University Nanfang Hospital

Ye Song ( $\nabla$ songye@smu.edu.cn )

Southern Medical University Nanfang Hospital

\section{Research}

Keywords: Glioma, ZEB2, miR-637, HMGA1, Vimentin, malignant progression

Posted Date: May 18th, 2020

DOl: https://doi.org/10.21203/rs.3.rs-28646/v1 
License: (c) (i) This work is licensed under a Creative Commons Attribution 4.0 International License. Read Full License

Version of Record: A version of this preprint was published at Molecular Therapy - Nucleic Acids on March 1st, 2021. See the published version at https://doi.org/10.1016/j.omtn.2020.12.029. 


\section{Abstract}

Background Glioma is the most common primary tumor of the central nervous system. We previously confirmed that zinc finger E-box binding homeobox (ZEB) 2 promotes the malignant progression of glioma, while microRNA-637 (miR-637) is associated with favorable prognosis in glioma. This study investigated the potential interaction between ZEB2 and miR-637 and its downstream signaling pathway in glioma. Methods Chromatin immunoprecipitation, luciferase reporter, and electrophoretic mobility shift assays were used to confirm ZEB2 binding to miR-637. Microarray and bioinformatic analyses were used to investigate targets of miR-637. MTT and EdU assays, transwell assays, and Boyden assays were utilized to assess cell viability, migration, and invasion, respectively. A subcutaneous xenograft model was utilized for analyzing the role of miR-637/high mobility group A1 (HMGA1) interaction in vivo. Immunohistochemistry staining of clinical samples and bioinformatic analysis of the Chinese Glioma Genome Atlas and The Cancer Genome Atlas databases were performed to further confirm the ZEB2/miR-637/HMGA1/vimentin axis. Results ZEB2 was bound directly bind to the E-box elements in the miR-637 promoter and promoted cell proliferation, migration, and invasion via miR-637 downregulation. Subsequent screening confirmed that HMGA1 was a direct target of miR-637, while miR-637 could propagate the malignant phenotype of glioma by suppressing HMGA1 both in vitro and in vivo. Furthermore, the interaction between cytoplasmic HMGA1 and vimentin was observed, and vimentin inhibition could abolish increased migration and invasion induced by HMGA1 overexpression. Notably, the ZEB2/miR-637/HMGA1/vimentin axis was also evident in glioma tissue samples and public glioma datasets, while both HMGA1 and vimentin were associated with an unfavorable prognosis in glioma. Additionally, upregulated HMGA1 and vimentin were found in isocitrate dehydrogenase (IDH) wild-type and $1 p / 19 q$ non-codeletion diffusely infiltrating glioma. Conclusions We identified an oncogenic ZEB2/miR-637/HMGA1 signaling axis targeting vimentin that promotes both migration and invasion in glioma. This not only extends the current understanding of miR-637 regulation in glioma, but also unravels a novel signaling pathway that integrates a transcriptional factor, microRNA, and a chromatin remodeling factor to modulate the malignant phenotype of glioma.

\section{Background}

Glioma is the most common primary brain tumor in adults. Glioblastoma (GBM) is the most malignant type of glioma, characterized by a high level of infiltration, therapeutic resistance, and poor prognosis with a modest median survival of 14.2 months, even under radiation plus temozolomide therapy (1). Genetic changes, including isocitrate dehydrogenase (IDH) $1 / 2$ mutation, $1 p / 19 q$ codeletion, and $0^{6}$ methylguanine-DNA methyltransferase (MGMT) promoter methylation have recently been accepted as crucial molecular characteristics for prognosis prediction and subgroup stratification (2-4). Besides these genetic modifications, overexpression of oncogenic protein-coding genes and non-coding RNAs (ncRNAs) (5) and dysregulated post-translational modification (6) can also modulate the malignant phenotype of glioma. Thus, insights into their potential prognostic value are crucial. 
Emerging evidence has indicated that ncRNAs, constituting a vast majority of the human transcriptome and previously considered as non-functional (7), could also regulate gene expression and are involved in the progression of a variety of cancers (8-10). As an important component of ncRNAs, microRNA could regulate oncogenesis and tumor progression by either blocking $\mathrm{mRNA}$ translation or promoting mRNA degradation $(11,12)$. We previously confirmed that microRNA-637 (miR-637) is a favorable prognosis marker in glioma that targets the 3区-untranslated region (UTR) of Akt1 (13). Further targets of miR-637, such as signal transducer and activator of transcription 3 (STAT3) and nuclear protein 1 (NUPR1) $(14,15)$, have been validated so far, but there remains a lack of knowledge regarding upstream regulation.

The zinc finger E-box binding homeobox (ZEB) family comprises two transcription factors, ZEB1 and ZEB2 (also known as SMAD-interacting protein-1 or SIP1), which both bind to E-box element CACCT(G) via zinc finger clusters and are critical regulators of cellular plasticity (16-18). In our previous study, we identified ZEB2 as a pivotal biomarker in glioma that promotes proliferation, migration, and invasion by inducing the epithelial-mesenchymal transition (EMT) process and cell cycle progression (19). A recent study showed that ZEB2 was inhibited by several miRNAs, such as miR-153 (20), miR-155 (21), and miR$30 \mathrm{a}$ (22), and these interactions significantly suppressed the EMT process. On the other hand, ZEB2 was also found to regulate miRNA expression as a repressive transcription factor. For example, there is a reciprocal interaction between ZEB2 and miR-203; while miR-203 inhibited ZEB2 through binding to its 3》UTR region, ZEB2 could directly abolish miR-203 expression by binding to an E-box element within the promoter of miR-203 (23). Although miR-637 has been identified as a negative regulator of the EMT process (24), its potential association with ZEB2 remains unknown.

Herein, the present study aimed to investigate the potential regulation of miR-637 by ZEB2 and explore its downstream signaling pathway in glioma. Our novel findings indicated that ZEB2 directly targets an Ebox element in the promoter region of miR-637 and inhibits its expression, leading to the promotion of the malignant phenotype of glioma through upregulating HGMA1, a downstream target of miR-637, both in vitro and in vivo. Moreover, vimentin was found to interact with cytoplasmic high mobility group A1 (HMGA1) and correlate with poor prognosis in glioma. This is the first report to demonstrate the upstream regulation of miR-637 and to identify the ZEB2/miR-637/HMGA1 oncogenic signaling axis as a critical regulator of the EMT process and glioma progression.

\section{Methods}

\section{Cell culture and clinical tissues collection}

The human glioma cell lines U251 and U87 and human embryo kidney 293T cells were purchased from the Chinese Academy of Sciences (Shanghai, China) and cultured in Dulbecco's modified Eagle's medium (DMEM; Hyclone, UT, USA) supplemented with $10 \%$ fetal calf serum (Hyclone) at $37^{\circ} \mathrm{C}$ in a humidified atmosphere of $5 \% \mathrm{CO}_{2}$. We obtained 69 paraffin-embedded glioma samples from the Department of Neurosurgery, Nanfang Hospital, Southern Medical University, Guangzhou, China. These glioma samples were from 40 male and 29 female patients aged 3-78 years (median age, 40 years). Of them, 65 were 
regularly and closely followed up, two lacked ZEB expression data, and nine lacked miR-637 expression data due to braking of the sectioned sample during the immunohistochemistry (IHC) or in situ hybridization (ISH) assays. Prior consent was obtained from all of the patients for the use of their clinical materials for research purposes, and approval was obtained from the Ethics Committees of Nanfang Hospital. All specimens had a confirmed pathological diagnosis and were classified according to the World Health Organization (WHO) criteria.

\section{Chromatin immunoprecipitation (ChIP) assay}

Protein-DNA complexes were immunoprecipitated from U251 and U87 cells using a Chromatin Immunoprecipitation Kit (Millipore, Billerica, MA, USA) according to the manufacturer's protocol with antiZEB2 (Bethyl Laboratories, Montgomery, TX, USA) and normal mouse IgG (Millipore) polyclonal antibodies, the latter serving as a control for nonspecific DNA binding. The precipitated DNA was then subjected to PCR with specific primers to amplify the putative ZEB2 binding region analyzed via electrophoresis (Supplementary Table 1).

\section{Real-time quantitative PCR (qPCR), PCR, and gel electrophoresis analysis}

RNA was extracted from glioma cells using Trizol reagent (Takara Bio Inc., Shiga, Japan). For qPCR, cDNA was amplified using SYBR ${ }^{\circledR}$ Green PCR Master Mix (Toyobo, Osaka, Japan) or miRNA PrimeScript RT Enzyme Mix (Takara Bio) and analyzed following gel electrophoresis. The relative expression level of mRNA or miRNA was normalized to $\beta$-actin or U6, respectively. The specific primers used for miR-637, HMGA1, and $\beta$-actin are shown in Supplementary Table 1.

\section{Luciferase reporter assay}

To confirm that miR-637 was a direct target of ZEB2, fragments of miR-637 promoter containing two different ZEB2 binding sites were cloned into pGL3-Basic vectors. These vectors were co-transfected with ZEB2 overexpression plasmid or small interfering RNA (siRNA) targeting ZEB2 into 293T cells in 48-well plates and then harvested for luciferase assay $48 \mathrm{~h}$ after transfection. The luciferase activity was determined using a Dual-Luciferase Reporter Assay System (Promega Corp, Fitchburg, WI, USA) according to the manufacturer's protocol.

To confirm that HMGA1 was a direct target of miR-637, a fragment of HMGA1 3囚-UTR containing the potential miR-637 binding sites was cloned into psiCHECK-2 vectors (named WT). Site-directed mutagenesis of the miR-637 binding site in the HMGA1 3区-UTR region was then performed using the GeneTailor Site-Directed Mutagenesis System (Invitrogen, Waltham, MA, USA; named Mut). Subsequently, WT or Mut vectors were co-transfected with miR-637 mimics or inhibitors into 293T cells in 48-well plates and then harvested for luciferase assay $48 \mathrm{~h}$ after transfection. Luciferase activity was determined, as mentioned above.

\section{Electrophoretic mobility shift assay (EMSA)}


We investigated ZEB2 binding activity on the promoter region of miR-637 using an EMSA Kit (Bioscience, Shanghai, China) according to the manufacturer's protocol. The samples with nuclear extracts from ZEB2-overexpressing glioma cells were used as positive controls, while samples without nuclear extracts were used as negative controls (NCs). For competition experiments, a specific oligonucleotide competitor (unlabeled wild-type ZEB2 site 1 probe, site $1 \mathrm{WT}$; unlabeled mutant ZEB2 site 1 probe, site 1 Mut) was added to the binding mixture $10 \mathrm{~min}$ before the addition of the labeled probe. Following electrophoresis, the bands were visualized on a BioSens Gel Imaging System (BIOTOP, Shanghai, China).

\section{Microarray analysis}

The total RNAs of U87 cells stably transfected with short hairpin RNA (shRNA) targeting ZEB2 and NC particles were isolated using Trizol reagent (Invitrogen) according to the manufacturer's instructions, respectively. After quality control checks, whole-genome expression microarray analysis (Affymetrix, Santa Clara, CA, USA) was performed by GeneChem Inc. (Shanghai, China). Genes in the ZEB2knockdown group with an absolute fold-change $>1.5$ and a $p$-value $<0.05$ compared with those in the NC group were considered as differentially expressed genes (DEGs).

\section{IHC and ISH assays}

Paraffin sections prepared from clinical samples and xenograft models were used for IHC or ISH assays to detect the expression levels of ZEB2, miR-637, HMGA1, vimentin, and Ki-67. For IHC staining, the indirect streptavidin-peroxidase method was used, as previously described (13). Following IHC staining, the tissue sections were examined and scored separately by two pathologists. The antibodies used were rabbit anti-ZEB2 (Cat. No. A302-473A, 1:500; Bethyl Laboratories), anti-HMGA1 (Cat. No. EPR7839, 1:200; Abcam, Cambridge, MA, USA), anti-vimentin (Cat. No. 10366-1-AP, 1:30; Proteintech, Rosemont, IL, USA), and anti-Ki67 (Cat. No. Ab16667, 1:100; Abcam). For the ISH assays, the tissue sections were hybridized with digoxigenin (DIG)-labeled miRCURY LNA probes (Bioscience) and were then incubated with alkalinephosphatase-conjugated anti-DIG Fab fragments. Finally, the sections were stained with BM purple alkaline phosphatase substrate (Roche, Switzerland) according to the manufacturer's instructions.

\section{Lentivirus production and transfection}

Lentiviral particles carrying hsa-miR-637 precursor vector, an expression construct for ZEB2 or HMGA1, or a counterpart NC sequence were constructed by GeneChem Inc. U251 and U87 cells were then transfected with the indicated lentiviral vector, and polyclonal cells with green fluorescent protein signals were selected for further experiments using fluorescence-activated cell sorting.

\section{Transfection with siRNAs, microRNA mimics/inhibitors, or plasmids}

siRNA targeting ZEB2, HMGA1 or vimentin, miR-637 mimics/inhibitors, or NC was designed and synthesized by RiboBio (Guangzhou, China). An expression plasmid for ZEB2 and HMGA1 was designed and synthesized by Vigenebio (Jinan, China). The efficiency was validated prior to the experiments by western blot or qPCR. Twelve h prior to transfection, U87 or U251 cells were plated into 6-well or 96-well 
plates (Wuxi NEST Biotechnology Co., Ltd., Jiangsu, China) at 30\%-50\% confluence. During transfection, Lipofectamine 2000 Transfection Reagent (Thermo Fisher Scientific, MA, USA) was used to facilitate the siRNA transfection into cells according to the manufacturer's protocol. The cells were collected after 48$72 \mathrm{~h}$ for further experiments. The specific sequences are shown in Supplementary Table 2.

\section{Cell viability assay}

Cell proliferation was analyzed using 3-(4,5-dimethylthiazol-2-yl)-2,5-diphenyltetrazolium bromide (MTT) assays. Briefly, cells were seeded into 96-well plates at a density of 1000-1500 cells/well and incubated for 1,2 , or 3 days. Approximately $20 \mu$ of MTT ( $5 \mathrm{mg} / \mathrm{ml}$; Sigma-Aldrich, St. Louis, MO, USA) was added into each well and incubated at $37^{\circ} \mathrm{C}$ in a $5 \% \mathrm{CO}_{2}$ atmosphere for $4 \mathrm{~h}$. At the end of the incubation, the supernatants were removed, and $150 \mu$ l of dimethyl sulfoxide (Sigma-Aldrich) was added to each well. The absorbance value (optical density) of each well was measured at $490 \mathrm{~nm}$ at the same time for the following 7 days. For each experimental condition, five wells were used for replicates.

\section{EdU incorporation assay}

Proliferating U251 and U87 cells were examined using the Cell-Light ${ }^{\mathrm{TM}}$ EdU Apollo ${ }^{\circledR} 488$ In Vitro Imaging Kit (RiboBio) according to the manufacturer's protocol. Briefly, the cells were incubated with $10 \mu \mathrm{M}$ of 5ethynyl-2X-deoxyuridine (EdU) for $2 \mathrm{~h}$ before fixation with $4 \%$ paraformaldehyde, permeabilization by $0.3 \%$ Triton X-100, and EdU staining. The nuclei were stained with $5 \mu \mathrm{g} / \mathrm{mL}$ DAPI for $10 \mathrm{~min}$. The number of EdU-positive cells was counted under a fluorescent microscope in five random fields (200x magnification). All assays were independently performed in triplicate.

\section{In vitro migration and invasion assays}

In vitro cell migration assays (transwell assay) and invasion assays (Boyden assay) were performed according to our previous study (13). For the cell migration assay, $1 \times 10^{4}$ cells in $100 \mu \mathrm{l}$ DMEM medium without fetal bovine serum (FBS) were seeded in the upper chamber of the transwell apparatus (Corning Costar, Corning, NY, USA), and $500 \mu$ l of DMEM with $10 \%$ FBS was added to the lower chamber as a chemoattractant. Following incubation for $6 \mathrm{~h}$ at $37^{\circ} \mathrm{C}$ in a $5 \% \mathrm{CO}_{2}$ atmosphere, the insert was washed with phosphate-buffered saline, and cells on the top surface of the insert were removed with a cotton swab. Cells adhering to the lower surface were fixed with methanol, stained with crystal violet solution, and counted under a microscope in five predetermined fields (200 x magnification). All assays were independently repeated at least three times. The procedure for the Boyden assay was similar to the transwell assay, except that the transwell apparatus was first precoated with $24 \mu \mathrm{g} / \mu \mathrm{l}$ of Matrigel (R\&D Systems, Minneapolis, MN, USA), and the cells were incubated for $12 \mathrm{~h}$ at $37^{\circ} \mathrm{C}$ in a $5 \% \mathrm{CO}_{2}$ atmosphere. Cells adhering to the lower surface of the insert were counted in the same manner as for the cell migration assay.

\section{Western blot analysis}


Western blot was carried out as previously described (19) with mouse polyclonal ZEB2 antibody (1:200; Santa Cruz Biotechnology, Inc., Dallas, TX, USA) and rabbit polyclonal HMGA1 (1:500; Abcam), Ncadherin, vimentin, and p15 antibodies (1:1000; Cell Signaling Technology, Danvers, MA, USA) as well as $\beta$-catenin and cyclin D1 antibodies (1:1000; Abcam). Mouse monoclonal $\beta$-actin antibody (1:1000; CoWin Biosciences, Beijing China) was used for normalization. An HRP-conjugated anti-rabbit or anti-mouse IgG antibody was used as the secondary antibody (1:2000; CoWin Biosciences). Signals were detected using enhanced chemiluminescence reagents (FdBio Science, Shenzhen, China).

\section{Subcutaneous xenograft model}

We subcutaneously injected $1 \times 10^{6}$ U87 cells transfected with shHMGA1 or the control lentiviral empty vector (mock; $n=5$ per group) in $100 \mu$ of DMEM without FBS into the dorsal flank areas of 6-week-old male BALB/c nude mice. The mice were sacrificed after 28 days, and the tumor tissues were excised and weighed. All mice were maintained in a barrier facility in racks of cages supplied with HEPA-filtered air and fed an autoclaved laboratory rodent diet. All animal studies were conducted in accordance with the principles and procedures outlined in the National Institutes of Health Guide for the Care and Use of Animals under assurance number A3873-1.

\section{Bioinformatic analysis}

Sequencing data and clinical data from the Chinese Glioma Genome Atlas (CGGA) and The Cancer Genome Atlas (TCGA) cohorts were retrieved from the CGGA database (http://www.cgga.org.cn) and the GEPIA database (http://gepia.cancer-pku.cn/index.html), respectively. The gene expression levels of different samples were measured in terms of fragments per kilobase of transcript per million in the CGGA cohort or transcript per million fragment in the TCGA cohort. Expression and survival analyses were subsequently performed. Putative mi-637 targets were predicted with three public databases: TargetScan 7.2 (http://www.targetscan.org/vert_72), miRDB (http://mirdb.org), and PITA

(https://genie.weizmann.ac.il/pubs/mir07), which were then intersected with the microarray-based gene signatures to confirm potential targets of miR-637.

\section{Statistical analysis}

All quantified data represented an average of at least triplicate samples. SPSS 22.0 (IBM Corp., Armonk, NY, USA) and GraphPad Prism 8.0 (GraphPad Software, San Diego, CA, USA) software were used for statistical analysis. Data are presented as mean \pm standard deviation. Two-way analysis of variance or two-tailed Student's $t$-test was used for comparisons between groups. Chi-square or Fischer's test was used to identify differences between categorical variables. Differences were considered statistically significant at $p$-values $<0.05$. All statistical tests were two-sided. Single, double, and triple asterisks in the figures indicate statistical significance $\left({ }^{\star} p<0.05,{ }^{\star} p<0.01\right.$ and $\left.{ }^{\star \star \star} p<0.001\right)$.

\section{Results}




\section{miR-637 is directly targeted by the transcriptional factor ZEB2 in glioma}

Two putative ZEB2 binding sites (CACCT) were found within the promoter region of miR-637 (Fig. 1A). To validate this result, we performed ChIP assays along with electrophoresis analysis using primers that specifically amplified these two regions. Significant enrichment of both putative binding regions was observed (Fig. 1B and C). To further support these results, luciferase assays were then performed using constructs containing ZEB2 binding site 1 or 2 downstream of a constitutively active luciferase reporter gene. In accordance with the ChIP assay results, transfection with ZEB2-expressing plasmid significantly decreased luciferase expression when it was coupled to the binding site 1 or site 2 construct, while knockdown of ZEB2 with siZEB2 led to increased luciferase activity (Fig. 1D). We further confirmed the ZEB2 binding site 1 via an EMSA in which no band shift was detected when the nuclear extract was incubated with the wild-type ZEB2 probes, while a band shift was observed when mutant ZEB site 1 probes were added (Supplementary Fig. 1).

Having confirmed that miR-637 was a direct target of ZEB2, we then examined their expression levels and correlation in clinical samples. Consistent with our previous results, a higher level of ZEB2 expression was detected in high-grade glioma (grade III and IV) compared with low-grade glioma (grade I and II), while a higher level of miR-637 expression was detected in low-grade glioma (Fig. 1E). A negative correlation between the expression level of miR-637 and ZEB2 was evident (Fig. 1F). Collectively, these results suggested that ZEB2 directly targeted an E-box element in the miR-637 promoter and might exert an inhibitory effect on miR-637 expression, although further evidence is needed.

\section{ZEB2 promotes the malignant phenotype of glioma by downregulating miR-637 in vitro}

ZEB2 is a critical transcription factor involved in the EMT process, and we previously confirmed that ZEB2 was associated with the malignant phenotype of glioma (19). In contrast, miR-637 was identified as a marker of favorable prognosis in glioma (13). Building on these results led to an assumption that ZEB2 could facilitate the malignant phenotype of glioma by directly suppressing miR-637. To prove this, we transfected U251 and U87 cells with shRNA targeting ZEB2 and found increased miR-637 levels under ZEB2 knockdown (Fig. 2A).

Having observed that ZEB2 suppressed miR-637 expression, we then investigated the potential role of ZEB2/miR-637 interaction in the malignant phenotype of glioma. Both the MTT assay (Fig. 2B) and EdU incorporation assay (Fig. $2 \mathrm{C}$ and D) revealed that ectopic miR-637 expression by lentiviral particles or miR-637 mimics could abolish the increased cell viability and proliferation mediated by ZEB2 overexpression in U251 and U87 cells. Similar results were observed in the case of migration and invasion. In transwell (Fig. 2E and F) and Boyden assays (Fig. 2G and H), while ectopic ZEB2 expression promoted the migratory and invasive activity of U251 and U87 cells, concomitant miR-637 overexpression significantly attenuated migration and invasion in glioma cells compared with ZEB2 overexpression alone (Fig. 2E-H). Collectively, ZEB2 promoted the malignant phenotype of glioma through transcriptional inhibition of miR-637. 
We then searched for potential target genes of miR-637 using the TargetScan, miRDB, and PITA databases to elucidate the molecular mechanism downstream of miR-637. Furthermore, we determined microarray-based gene expression signatures under ZEB2 knockdown compared with an NC, and genes with an absolute fold-change $>1.5$ and $p<0.05$ were considered as DEGs. Since these genes might be indirect targets of ZEB2 via miR-637, the intersection between the downregulated genes under ZEB2 knockdown and the potential miR-637 target genes was examined. We found 25 genes with overlapping microarray and bioinformatic results (Fig. 3A). In our previous study, we had already confirmed that ZEB2 was involved in the EMT process as well as in the cell cycle and apoptosis (19). A literature search led us to postulate that the PI3K/Akt signaling pathway could be a candidate downstream of ZEB2 due to its pleiotropic effects in glioma (25-27) and because Akt1 was previously confirmed as a direct target of miR-637 (19).

Therefore, we expanded our findings, focusing on HMGA1 protein, which is a crucial activator of the PI3K/Akt pathway (28). Both qPCR analysis (Fig. 3B) and western blot (Fig. 3C) revealed that ectopic miR-637 expression with miR-637 mimics significantly decreased the HMGA1 expression level.

Furthermore, putative binding sites for miR-637 were predicted in the 3囚-UTR region of HMGA1, and a luciferase reporter vector was then constructed with the target region sequence (HMGA1 wild-type 3囚UTR) or a mutant target region sequence (HMGA1 mutant 3囚-UTR) (Fig. 3D). The luciferase reporter assay indicated that the co-transfection of miR-637 mimics significantly decreased the luciferase activity of the HMGA1 wild-type 3区-UTR construct, while miR-637 inhibitor led to increased luciferase activity (Fig. 3E, column 1 and 2). A mutation of the putative binding sequence could successfully abrogate these effects (Fig. 3E, column 3 and 4).

Consistently, while a higher level of HMGA1 expression was identified in high-grade glioma (Fig. 3F), a negative correlation between HMGA1 expression and miR-637 expression was found (Fig. 3G). Collectively, our results indicated that miR-637 directly targeted HMGA1 and inhibited its expression.

\section{miR-637 suppresses the malignant phenotype of glioma by downregulating HMGA1 in vitro}

Although HMGA1 was confirmed as a downstream target of miR-637, the effect of their interaction on the malignant phenotype of glioma remained unelucidated. Therefore, an MTT assay was first performed to evaluate their effects on cell viability, revealing that ectopic HMGA1 expression could successfully rescue the suppressed cell proliferation due to miR-637 overexpression in U251 and U87 cells (Fig. 4A).

Consistent with this, the EdU incorporation assay demonstrated an increased rate of cell proliferation in the rescued group compared with treatment with miR-637 mimics alone (Fig. 4B and C). Regarding cell migration and invasion, in both U251 and U87 cells, the inhibited cell migration and invasion resulting from miR-637 overexpression were significantly rescued by ectopic HMGA1 expression (Fig. 4D-G). Briefly, these results confirmed that miR-637 suppressed the malignant phenotype of glioma, including cell proliferation, migration, and invasion, by downregulating HMGA1 in vitro.

\section{miR-637 modulates xenograft tumor growth by targeting HMGA1 in vivo}


To further interrogate HMGA1 as a direct target mediating the biological effects of miR-637, we established a subcutaneous xenograft model to examine the relationship between miR-637 and HGMA1 in a more biologically relevant setting. U87/NC, U87/HMGA1, U87/miR-637, and U87/miR-637/HMGA1 cells were subcutaneously implanted into nude mice, respectively. The mice were sacrificed at 28 days after tumor implantation, and the tumors were weighed. U87/HMGA1 cells formed larger tumors than U87/NC cells (Fig. 5A, panel 1 and 2), while the U87/miR-637/HMGA1 group exhibited larger tumors than the U87/miR-637 group (Fig. 5A, panel 3 and 4). Greater tumor weight was also evident in the U87/miR$637 / H M G A 1$ group compared with that in the U87/miR-637 group, although this difference was not statistically significant (Fig. 5B). We then evaluated the protein expression level of cell proliferation marker $\mathrm{Ki}-67$ in these tumors. Consistently, a higher proliferative rate in the U87/HMGA1 group compared with that in the U87/NC group was confirmed, whereas a significantly higher proliferative rate was observed in the U87/miR-637/HMGA1 group compared with that in the in U87/miR-637 group (Fig. 5C and D). Briefly, miR-637 suppressed xenograft tumor growth by targeting HMGA1 and then inhibiting the rate of cell proliferation in vivo.

\section{HMGA1 promotes glioma migration and invasion of through vimentin in vitro}

HMGA1 could regulate GBM cell stemness by modifying chromatin architecture at the promoter of SOX2, one of the major regulators of cell stemness (29). It was also reported that HMGA1 promoted tumor cell stemness and the EMT process in breast cancer (30). Consistently, we found significantly decreased levels of N-cadherin, vimentin, and cyclin D1 following HGMA1 knockdown in both U251 and U87 cells, while an increased level of p15 expression was observed (Fig. 6A). Interestingly, the anti-HGMA1 CoIP assay demonstrated that vimentin, an intermediate filament expressed in immature astrocytes, could directly bind to HMGA1 (Fig. 6B, panel 1). Reciprocal immunoprecipitation with anti-vimentin antibody similarly indicated this interaction (Fig. 6B, panel 2). In glioma tissue samples, the level of vimentin expression was higher in high-grade glioma (Fig. 6C), whereas a positive correlation was found between the expression levels of vimentin and HMGA1 (Fig. 6D). A correlation analysis based on the CGGA as well as the TCGA glioma cohorts further supported our result (Supplementary Fig. 2). Vimentin is a critical member of the family of intermediate filaments and is involved in cell contraction and migration (31). Thus, we then focused on examining glioma cell migration and invasion. While HMGA1 significantly promoted both migration and invasion in vitro, concomitant inhibition of vimentin could successfully abolish this effect (Fig. 6E-H). Collectively, our results indicated that HMGA1 could interact with vimentin and facilitate both migration and invasion through vimentin in glioma.

\section{HMGA1 and vimentin are associated with unfavorable prognosis in glioma}

This oncogenic ZEB2/miR-637/HMGA1/vimentin signaling pathway was further confirmed with a cohort of 69 prospectively collected primary glioma tissues from our department. Since vimentin is an intermediate filament expressed in astrocytic progenitors, a high level of vimentin expression was observed in astrocytoma samples. There was no significant association between the expression level of vimentin or HMGA1 and the patient's age or gender (Table 1). Notably, higher expression levels of ZEB2, HGMA1, and vimentin were observed in high-grade glioma (grade III and IV) compared with low-grade 
glioma (grade I and II), while a lower expression level of miR-637 was observed in high-grade glioma (Table 1). Interestingly, in the CGGA glioma cohort, we found that HMGA1 and vimentin were both markedly upregulated in IDH wild-type and 1p/19q non-codeletion glioma (Fig. 7A-D), which is considered a diffusely infiltrating glioma according to the 2016 WHO central nervous system (CNS) tumor classification (42).

lation between the clinicopathological factors and expression of ZEB2/miR-637/HMGA1/Vimentin axis

\begin{tabular}{|c|c|c|c|c|c|c|c|c|c|c|c|c|c|}
\hline \multirow[t]{2}{*}{ s } & \multirow[t]{2}{*}{$\mathbf{n}$} & \multicolumn{3}{|c|}{ ZEB2 expression } & \multicolumn{3}{|c|}{ miR-637 expression } & \multicolumn{3}{|c|}{ HMGA1 expression } & \multicolumn{3}{|c|}{ Vimentin expression } \\
\hline & & High & Low & $p$ & High & Low & $p$ & High & Low & $p$ & High & Low & $p$ \\
\hline & 40 & 17 & 22 & 0.286 & 14 & 21 & 0.12 & 20 & 20 & 0.45 & 19 & 21 & 0.35 \\
\hline & 29 & 14 & 14 & & 13 & 12 & & 13 & 16 & & 16 & 13 & \\
\hline & 18 & 9 & 9 & 0.336 & 5 & 9 & 0.163 & 10 & 8 & 0.24 & 11 & 7 & 0.11 \\
\hline & 51 & 22 & 27 & & 22 & 24 & & 23 & 28 & & 24 & 27 & \\
\hline \multicolumn{14}{|c|}{ pe } \\
\hline & 46 & 21 & 23 & 0.279 & 18 & 21 & 0.213 & 22 & 24 & 0.10 & 24 & 22 & 0.03 \\
\hline & 15 & 6 & 9 & & 5 & 8 & & 5 & 10 & & 4 & 11 & \\
\hline & 8 & 4 & 4 & & 4 & 4 & & 6 & 2 & & 7 & 1 & \\
\hline & 34 & 4 & 29 & $<0.001$ & 24 & 8 & $<0.001$ & 8 & 26 & $<0.001$ & 8 & 26 & $<0.001$ \\
\hline & 35 & 27 & 7 & & 3 & 25 & & 25 & 10 & & 27 & 8 & \\
\hline
\end{tabular}

$\mathrm{AT}$, astrocytic tumor; OT, oligodendrogial tumor; WHO, world health organization.

We have already confirmed that ZEB2 is a potential marker for poor prognosis in glioma (19), whereas miR-637 is correlated with favorable prognosis in glioma (13). Furthermore, a Kaplan-Meier analysis of overall survival in our cohort revealed that glioma patients with higher HMGA1 or vimentin expression exhibited poorer prognosis (Fig. 7E and H), although the differences between the high HMGA1 expression group and low HMGA1 expression group were not statistically significant. We further validated these results on a larger scale with the CGGA and TCGA databases, and similar results were found (Fig. 7F and I, Fig. $7 \mathrm{G}$ and J, respectively). Taken together, both HGMA1 and vimentin were associated with an aggressive growth pattern and poor prognosis in glioma, while the oncogenic ZEB2/miR$637 /$ HMGA1/vimentin signaling pathway was evident in the glioma samples.

\section{Discussion}

To date, the engagement and regulation of ncRNAs remain inadequately characterized, including the potential interaction between protein-coding genes and ncRNAs as well as interactions among ncRNAs. A reciprocal inhibition between ZEB2 and miR-203 that regulates cancer stem cell growth was found in breast cancer (32), while ZEB2 was found to be downregulated under transforming growth factor- $\beta$ - 
induced miR-192 overexpression, leading to p53 upregulation (33). Our previous study revealed that ZEB2 was involved in the malignant progression of glioma (19), and miR-637 was a favorable prognosis marker in glioma (13), but the relationship between ZEB2 and miR-637 was unelucidated. In this study, our findings built upon the current knowledge of miR-637 regulation by revealing that ZEB2 could directly bind to the promoter of miR-637 and repress its expression, which further led to the upregulation of HMGA1, a novel downstream target of miR-637. Targeting the EMT process, HMGA1 could further interact with vimentin and promote glioma migration and invasion (Fig. 9).

MiR-637 plays a pivotal role in a variety of cancers. It functions as a tumor suppressor via targeting and downregulating NUPR1 expression, which was shown to lead to the inhibition of proliferation, migration, and invasion in colorectal cancer cells (34). Moreover, supporting our previous results, miR-637 was found to target Akt1 and hamper tumorigenesis in both thyroid carcinoma and pancreatic ductal adenocarcinoma $(35,36)$. MiR-637 could also inhibit Akt phosphorylation and promote melanoma progression (37). Remarkably, although several circular RNAs or long non-coding RNAs (IncRNAs) were found to regulate miR-637 through the mechanism of competitive endogenous RNA, few studies have investigated the crosstalk between transcription factors and miR-637. Our results indicated that the critical EMT regulator ZEB2 could directly bind to the miR-637 promoter and suppress its expression, expanding the current understanding of miRNA regulation. Furthermore, HMGA1 was confirmed as another downstream target of miR-637, forming a complete regulatory network around miR-637.

HMGA1 is a member of the HMGA protein family, which does not possess intrinsic transcriptional activity but alters the chromatin structure by direct recognition of the A/T-rich sequences in the promoter and enhancer regions of multiple genes (38). HMGA1 has been proved to be involved in the regulation of stemness and the progression of glioma via a variety of mechanisms. For instance, HMGA1 was found to function as a critical regulator of GBM cell stemness by modifying chromatin architecture at the promoter of SOX2, and this effect was downstream of miR-296-5p (29). Moreover, the downregulation of IncRNA HIF1A-AS2 could impair the expression of HMGA1 and lead to inhibited growth of mesenchymal GBM stem-like cells (39). In accordance with a previous report (40), we found that HMGA1 was a key regulator of the EMT process and confirmed the interaction between HMGA1 and vimentin. Although HMGA1 is mostly reported as located in the nucleus, it was suggested that HMGA1 could be secreted through cytoplasmic translocation, and its cytoplasmic translocation could predict the aggressiveness of breast cancer (41). Supporting this result, our results further revealed that nuclear protein HMGA1 could be translocated into the cytoplasm and promoted the migration and invasion of glioma through interaction with vimentin, although further study on the precise mechanism of cytoplasmic translocation of HMGA1 is necessary.

Molecular features, such as IDH1/2 mutation and 1p/19q codeletion, were integrated with the histological appearance for a more accurate classification in the 2016 WHO CNS tumor classification (42). For example, diffuse astrocytoma and diffuse oligodendroglioma are grouped together based not only on their diffusely infiltrative growth pattern but also on their lack of IDH1/2 mutation (42). In IDH1 wild-type grade II glioma, intense expression of vimentin, an intermediate filament expressed in immature 
astrocytes, was observed, indicating the potential involvement of neural progenitors in IDH1 wild-type glioma (43). Oligodendroglioma characterized by whole-arm loss of $1 p$ and $19 q$ ( $1 p / 19 q$ codeletion) was associated with better prognosis, although its association with HMGA1 and vimentin is unclear. Our subgroup analysis revealed that HMGA1 and vimentin were overexpressed in astrocytoma with an intact $1 p / 19 q$ loci, indicating that further insights into gliomagenesis considering different origins are needed.

\section{Conclusions}

In conclusion, we are the first to report that miR-637 was directly inhibited by transcription factor ZEB2, while miR-637 could further suppress HMGA1, which interacts with downstream vimentin. Based on these findings, an oncogenic ZEB2/miR-637/HMGA1 signaling axis targeting vimentin was identified, which propagates both migration and invasion in glioma. Our study may shed new light on the integrated modulation of the malignant phenotype of glioma involving a transcription factor, microRNA, and a chromatin remodeling factor and facilitate a better understanding of glioma progression.

\section{Abbreviations}

GBM: glioblastoma; ncRNA: Non-coding RNAs; HMGA1: High mobility group protein A1; ZEB2: Zinc finger E-box-binding homeobox 2; DMEM: Dulbecco's modified Eagle's medium; ChIP: Chromatin immunoprecipitation; qPCR: Real-time Quantitative PCR; EMSA: Electrophoretic mobility shift assay; IHC staining: Immunohistochemical staining; siRNA: Small-interfering RNA; MTT: 3-(4,5-dimethylthiazol-2yl)-2,5-diphenyltetrazolium bromide; FBS: fetal bovine serum; CGGA: Chinese Glioma Genome Atlas; TCGA: The Cancer Genome Atlas; GEPIA: Gene Expression Profiling Interactive Analysis.

\section{Declarations}

\section{Ethics approval and consent to participate}

Clinical sample collection and animal experiments were performed under the approval of the ethics committee of Nanfang Hospital, Southern Medical University.

\section{Consent for publication}

Not applicable.

\section{Availability of data and materials}

The databases analyzed in the present study are available in CGGA database (http://www.cgga.org.cn), GEPIA database (http://gepia.cancer-pku.cn/index.html), TargetScan v7.2 (http://www.targetscan.org/vert_72), miRDB (http://mirdb.org) and PITA (https://genie.weizmann.ac.il/pubs/mir07).

\section{Competing interest}




\section{The authors have declared no competing interest.}

\section{Author's contribution}

YS designed and supervised the study; $Y Z, T Q$ and JL performed most of experiments and statistical analysis; $A X$ and CX performed manuscript writing; JL, SD and ZZ performed ChIP, luciferase reporter assays, and EMSA assay; $\mathrm{HL}$ and $\mathrm{XZ}$ performed figures editing. All authors have read and approved the final manuscript.

\section{Acknowledgements}

This study was supported by National Nature Science Fund of China (NO. 81702473, 81760450, 81872064), Natural Science Fund of Guangdong Province, China (NO. 2016A030313549), President Fund of Nanfang Hospital (NO. 2016C019), Science and Technology Program of Guangzhou, China (NO. 201607010350), Natural Science Fund of Tibet Autonomous Region(NO. XZ2017ZR-ZYZ27), Knowledge Innovation Program of Shenzhen (NO. JCYJ20170307110825922). The funders had no role in study design, data collection, data analysis, decision to publish, or preparation of the manuscript. The authors would also like to thank Enago (www.enago.cn) for the English language review.

\section{References}

1. Johnson DR, O'Neill BP. Glioblastoma survival in the United States before and during the temozolomide era. Journal of neuro-oncology. 2012;107(2):359-64.

2. Brito C, Azevedo A, Esteves S, Marques AR, Martins C, Costa I, et al. Clinical insights gained by refining the 2016 WHO classification of diffuse gliomas with: EGFR amplification, TERT mutations, PTEN deletion and MGMT methylation. BMC Cancer. 2019;19(1):968.

3. Bund C, Guergova-Kuras M, Cicek AE, Moussallieh FM, Dali-Youcef N, Piotto M, et al. An integrated genomic and metabolomic approach for defining survival time in adult oligodendrogliomas patients. Metabolomics. 2019;15(5):69.

4. Harat M, Blok M, Harat A, Soszynska K. The impact of adjuvant radiotherapy on molecular prognostic markers in gliomas. Onco Targets Ther. 2019;12:2215-24.

5. Reon BJ, Anaya J, Zhang Y, Mandell J, Purow B, Abounader R, et al. Expression of IncRNAs in LowGrade Gliomas and Glioblastoma Multiforme: An In Silico Analysis. PLoS Med. 2016;13(12):e1002192.

6. Yin J, Park G, Lee JE, Choi EY, Park JY, Kim TH, et al. DEAD-box RNA helicase DDX23 modulates glioma malignancy via elevating miR-21 biogenesis. Brain. 2015;138(Pt 9):2553-70.

7. Kent OA, Steenbergen C, Das S. In Vivo Nanovector Delivery of a Heart-specific MicroRNA-sponge. Journal of visualized experiments: JoVE. 2018(136).

8. Song H, Li D, Wu T, Xie D, Hua K, Hu J, et al. MicroRNA-301b promotes cell proliferation and apoptosis resistance in triple-negative breast cancer by targeting CYLD. BMB Rep. 2018;51(11):602- 
7.

9. Wang Q, Cai J, Fang C, Yang C, Zhou J, Tan Y, et al. Mesenchymal glioblastoma constitutes a major ceRNA signature in the TGF-beta pathway. Theranostics. 2018;8(17):4733-49.

10. Wang X, Ding Y, Da B, Fei Y, Feng G. Identification of potential prognostic long noncoding RNA signatures based on a competing endogenous RNA network in lung adenocarcinoma. Oncol Rep. 2018;40(6):3199-212.

11. Ding L, Lan Z, Xiong X, Ao H, Feng Y, Gu H, et al. The Dual Role of MicroRNAs in Colorectal Cancer Progression. International journal of molecular sciences. 2018;19(9).

12. Huang Y. The novel regulatory role of IncRNA-miRNA-mRNA axis in cardiovascular diseases. J Cell Mol Med. 2018;22(12):5768-75.

13. Que T, Song Y, Liu Z, Zheng S, Long H, Li Z, et al. Decreased miRNA-637 is an unfavorable prognosis marker and promotes glioma cell growth, migration and invasion via direct targeting Akt1. Oncogene. 2015;34(38):4952-63.

14. Huang W, Cao J, Peng X. LINC01234 facilitates growth and invasiveness of oral squamous cell carcinoma through regulating the miR-637/NUPR1 axis. Biomed Pharmacother. 2019;120:109507.

15. Zhang Y, Li C, Liu X, Wang Y, Zhao R, Yang Y, et al. circHIPK3 promotes oxaliplatin-resistance in colorectal cancer through autophagy by sponging miR-637. EBioMedicine. 2019;48:277-88.

16. Hill L, Browne G, Tulchinsky E. ZEB/miR-200 feedback loop: at the crossroads of signal transduction in cancer. Int J Cancer. 2013;132(4):745-54.

17. Drapela S, Bouchal J, Jolly MK, Culig Z, Soucek K. ZEB1: A Critical Regulator of Cell Plasticity, DNA Damage Response, and Therapy Resistance. Front Mol Biosci. 2020;7:36.

18. Nam EH, Lee Y, Park YK, Lee JW, Kim S. ZEB2 upregulates integrin alpha5 expression through cooperation with Sp1 to induce invasion during epithelial-mesenchymal transition of human cancer cells. Carcinogenesis. 2012;33(3):563-71.

19. Qi S, Song Y, Peng Y, Wang H, Long H, Yu X, et al. ZEB2 mediates multiple pathways regulating cell proliferation, migration, invasion, and apoptosis in glioma. PloS one. 2012;7(6):e38842.

20. Zhou J, Xie M, Shi Y, Luo B, Gong G, Li J, et al. MicroRNA-153 functions as a tumor suppressor by targeting SET7 and ZEB2 in ovarian cancer cells. Oncol Rep. 2015;34(1):111-20.

21. Brown CY, Dayan S, Wong SW, Kaczmarek A, Hope CM, Pederson SM, et al. FOXP3 and miR-155 cooperate to control the invasive potential of human breast cancer cells by down regulating ZEB2 independently of ZEB1. Oncotarget. 2018;9(45):27708-27.

22. di Gennaro A, Damiano V, Brisotto G, Armellin M, Perin T, Zucchetto A, et al. A p53/miR-30a/ZEB2 axis controls triple negative breast cancer aggressiveness. Cell death differentiation. 2018;25(12):2165-80.

23. Duan X, Fu Z, Gao L, Zhou J, Deng X, Luo X, et al. Direct interaction between miR-203 and ZEB2 suppresses epithelial-mesenchymal transition signaling and reduces lung adenocarcinoma chemoresistance. Acta Biochim Biophys Sin. 2016;48(11):1042-9. 
24. Zhang Q, Wang G, Xu L, Yao Z, Song L. Long non-coding RNA LINC00473 promotes glioma cells proliferation and invasion by impairing miR-637/CDK6 axis. Artif Cells Nanomed Biotechnol. 2019;47(1):3896-903.

25. Yu M, Xue Y, Zheng J, Liu X, Yu H, Liu L, et al. Linc00152 promotes malignant progression of glioma stem cells by regulating miR-103a-3p/FEZF1/CDC25A pathway. Mol Cancer. 2017;16(1):110.

26. Koul D, Shen R, Kim YW, Kondo Y, Lu Y, Bankson J, et al. Cellular and in vivo activity of a novel PI3K inhibitor, PX-866, against human glioblastoma. Neurooncology. 2010;12(6):559-69.

27. Kaushik NK, Kaushik N, Yoo KC, Uddin N, Kim JS, Lee SJ, et al. Low doses of PEG-coated gold nanoparticles sensitize solid tumors to cold plasma by blocking the PI3K/AKT-driven signaling axis to suppress cellular transformation by inhibiting growth and EMT. Biomaterials. 2016;87:118-30.

28. Wei F, Zhang T, Deng SC, Wei JC, Yang P, Wang Q, et al. PD-L1 promotes colorectal cancer stem cell expansion by activating HMGA1-dependent signaling pathways. Cancer letters. 2019;450:1-13.

29. Lopez-Bertoni H, Lal B, Michelson N, Guerrero-Cazares H, Quinones-Hinojosa A, Li Y, et al. Epigenetic modulation of a miR-296-5p:HMGA1 axis regulates Sox2 expression and glioblastoma stem cells. Oncogene. 2016;35(37):4903-13.

30. Pegoraro S, Ros G, Piazza S, Sommaggio R, Ciani Y, Rosato A, et al. HMGA1 promotes metastatic processes in basal-like breast cancer regulating EMT and stemness. Oncotarget. 2013;4(8):1293308.

31. Li M, Zhang B, Sun B, Wang X, Ban X, Sun T, et al. A novel function for vimentin: the potential biomarker for predicting melanoma hematogenous metastasis. J Exp Clin Cancer Res. 2010;29:109.

32. Polytarchou $C$, lliopoulos D, Struhl K. An integrated transcriptional regulatory circuit that reinforces the breast cancer stem cell state. Proc Natl Acad Sci USA. 2012;109(36):14470-5.

33. Deshpande SD, Putta S, Wang M, Lai JY, Bitzer M, Nelson RG, et al. Transforming growth factor-betainduced cross talk between p53 and a microRNA in the pathogenesis of diabetic nephropathy. Diabetes. 2013;62(9):3151-62.

34. Wang L, Jiang F, Xia X, Zhang B. LncRNA FAL1 promotes carcinogenesis by regulation of miR637/NUPR1 pathway in colorectal cancer. Int J Biochem Cell Biol. 2019;106:46-56.

35. Xu RL, He W, Tang J, Guo W, Zhuang P, Wang CQ, et al. Primate-specific miRNA-637 inhibited tumorigenesis in human pancreatic ductal adenocarcinoma cells by suppressing Akt1 expression. Experimental cell research. 2018;363(2):310-4.

36. Yuan Q, Liu Y, Fan Y, Liu Z, Wang X, Jia M, et al. LncRNA HOTTIP promotes papillary thyroid carcinoma cell proliferation, invasion and migration by regulating miR-637. Int J Biochem Cell Biol. 2018;98:1-9.

37. Zhang J, Liu WL, Zhang L, Ge R, He F, Gao TY, et al. MiR-637 suppresses melanoma progression through directly targeting P-REX2a and inhibiting PTEN/AKT signaling pathway. Cellular and molecular biology (Noisy-le-Grand. France). 2018;64(11):50-7.

38. Pang B, Fan H, Zhang IY, Liu B, Feng B, Meng L, et al. HMGA1 expression in human gliomas and its correlation with tumor proliferation, invasion and angiogenesis. Journal of neuro-oncology. 
2012;106(3):543-9.

39. Mineo M, Ricklefs F, Rooj AK, Lyons SM, Ivanov P, Ansari KI, et al. The Long Non-coding RNA HIF1AAS2 Facilitates the Maintenance of Mesenchymal Glioblastoma Stem-like Cells in Hypoxic Niches. Cell reports. 2016;15(11):2500-9.

40. J W, B L, Y S, X W, J B, L Y. Exosome-Mediated Transfer of IncRNA HOTTIP Promotes Cisplatin Resistance in Gastric Cancer Cells by Regulating HMGA1/miR-218 Axis. OncoTargets and therapy. 2019;12:11325-38.

41. O M, C VP. S, M P, Y F, I A, et al. Extracellular HMGA1 Promotes Tumor Invasion and Metastasis in Triple-Negative Breast Cancer. Clinical cancer research: an official journal of the American Association for Cancer Research. 2018;24(24):6367-82.

42. DN L, A P, vD GRA, D F-B WKC, et al. The 2016 World Health Organization Classification of Tumors of the Central Nervous System: a summary. Acta Neuropathol. 2016;131(6):803-20.

43. Compes P, Tabouret E, Etcheverry A, Colin C, Appay R, Cordier N, et al. Neuro-radiological characteristics of adult diffuse grade II and III insular gliomas classified according to WHO 2016. Journal of neuro-oncology. 2019;142(3):511-20.

\section{Figures}

A

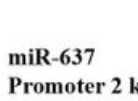

Promoter 2 kb

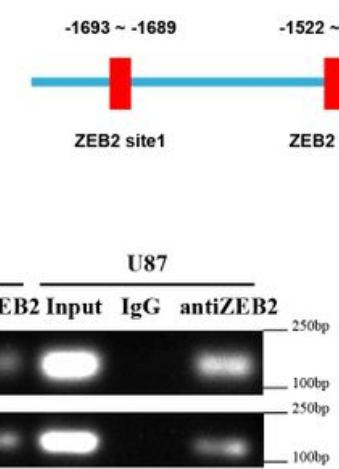

Putative ZEB2 binding sites in miR-637 promoter

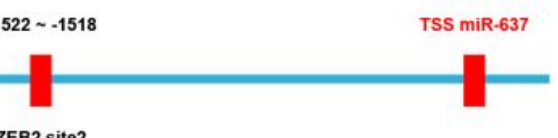

C

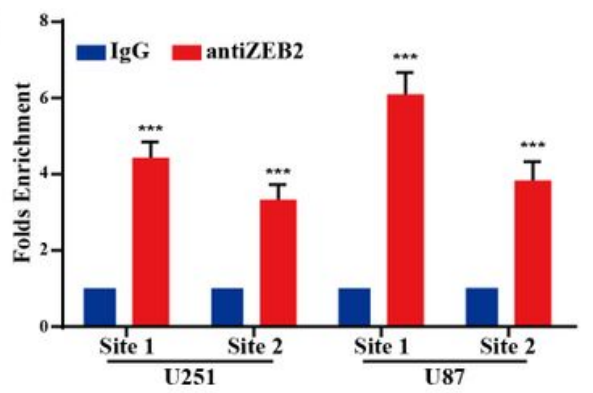

D

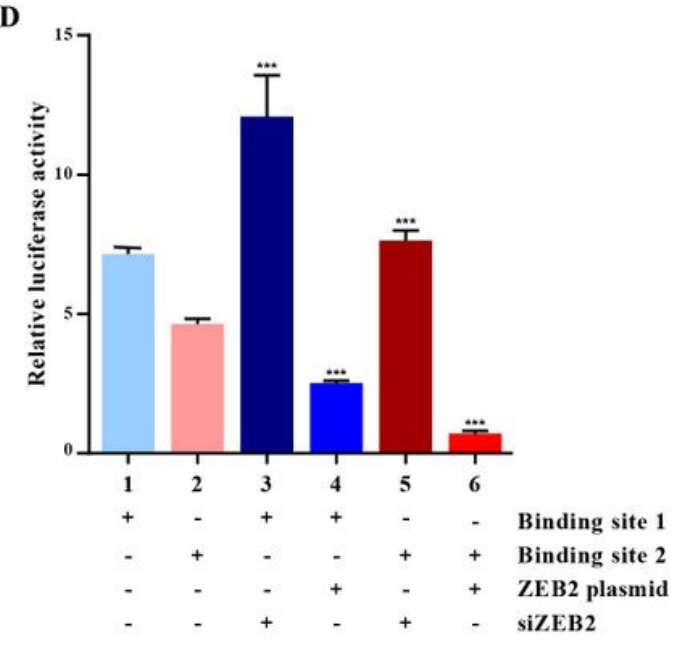

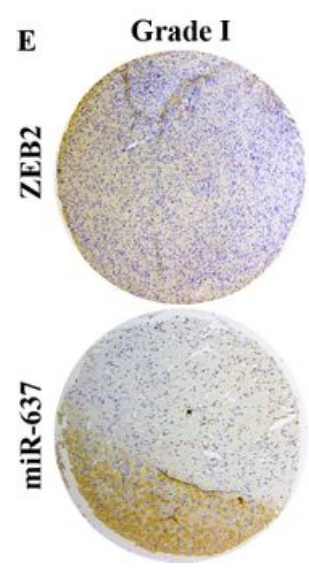

Grade II

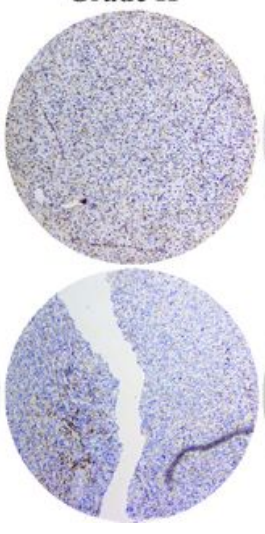

Grade III

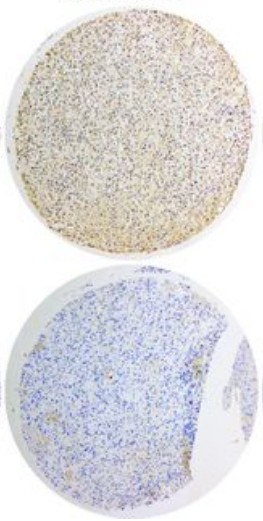

Grade IV

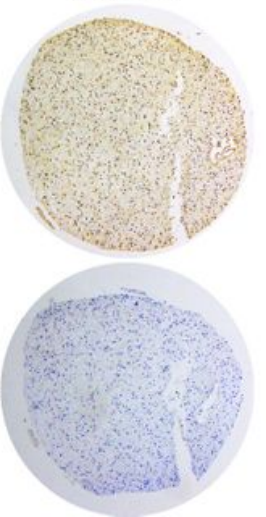

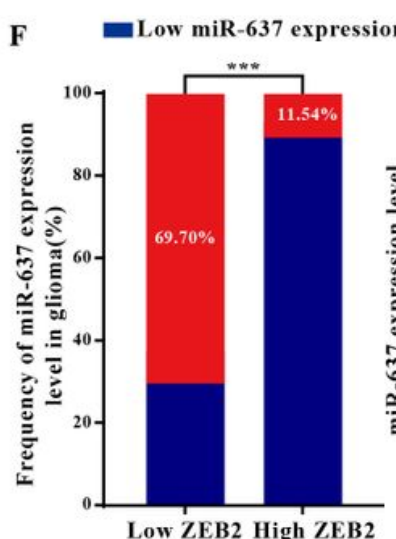

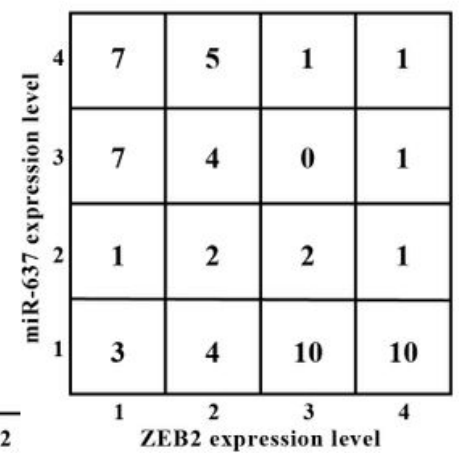




\section{Figure 1}

miR-637 is directly targeted by the transcriptional factor ZEB2 in glioma. (A) Schematic illustration of the two putative ZEB2-binding sites in the promoter region of miR-637. (B and C) ChIP assays along with electrophoresis analysis demonstrated amplification of both binding sites 1 and 2. (D) Relative luciferase activity of the indicated constructs was examined after the co-transfection of the ZEB2 plasmid or siZEB2 in 293 T cells. (E) IHC analysis of ZEB2 and ISH analysis of miR-637 in glioma tissues were performed. (F) Expression of miR-637 was negatively correlated with that of ZEB2 in glioma tissues. Data were presented as mean \pm S.D. $\left({ }^{*} p<0.05,{ }^{* *} p<0.01\right.$ and $\left.{ }^{* \star *} p<0.001\right)$. 


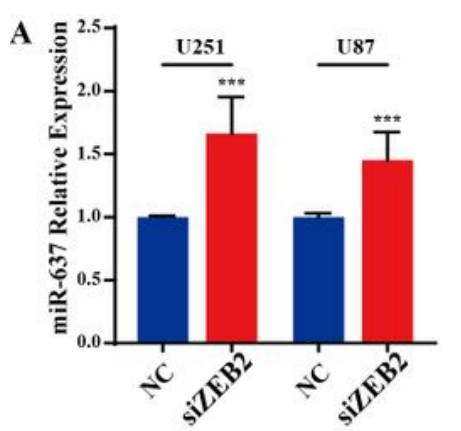

C

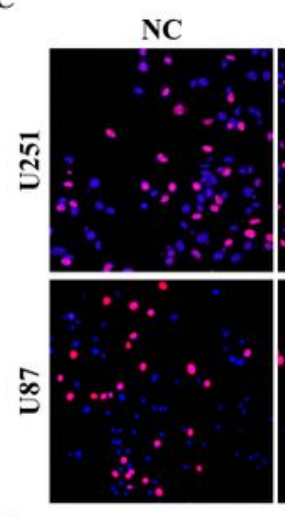

ZEB2 OE

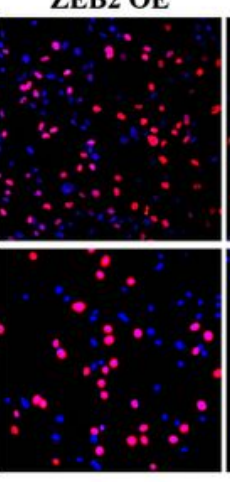

$\mathbf{E}$

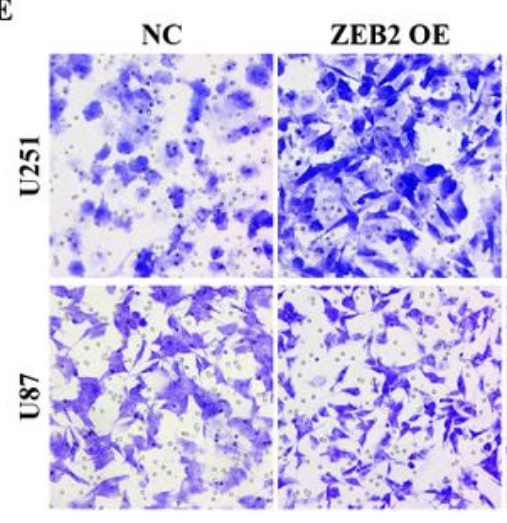

G

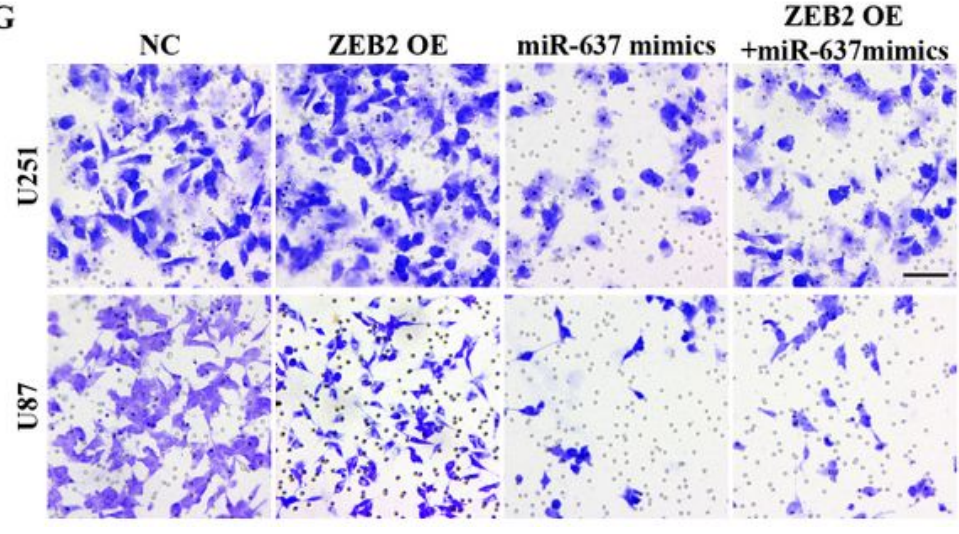
miR-637 mimics +miR-637mimics

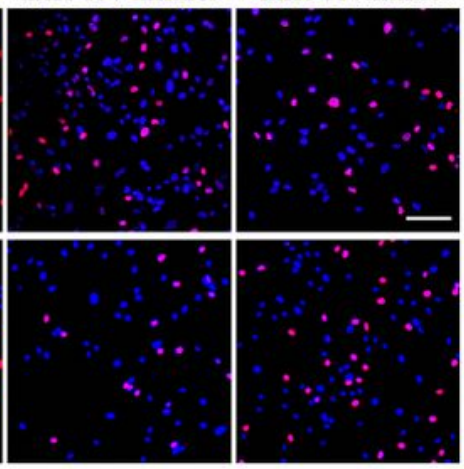

ZEB2 OE miR-637 mimics +miR-637mimics
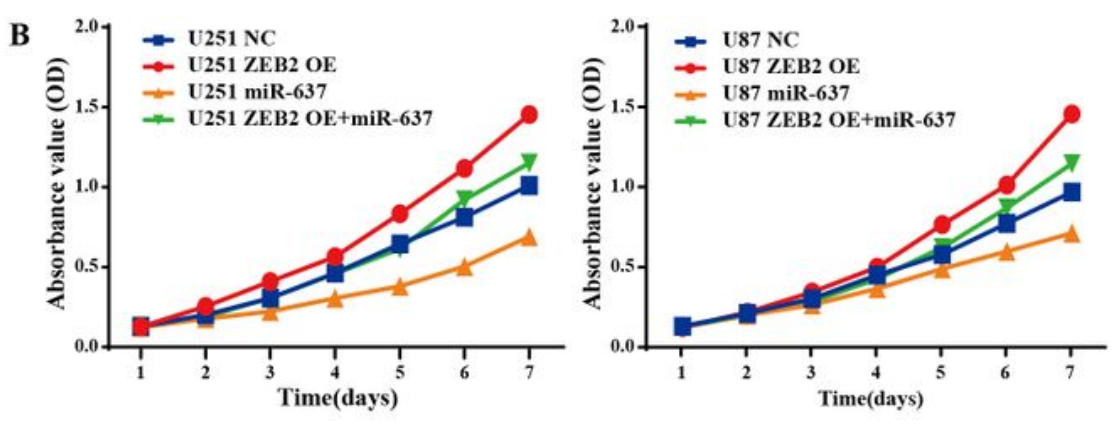

ZEB2 OE D

F
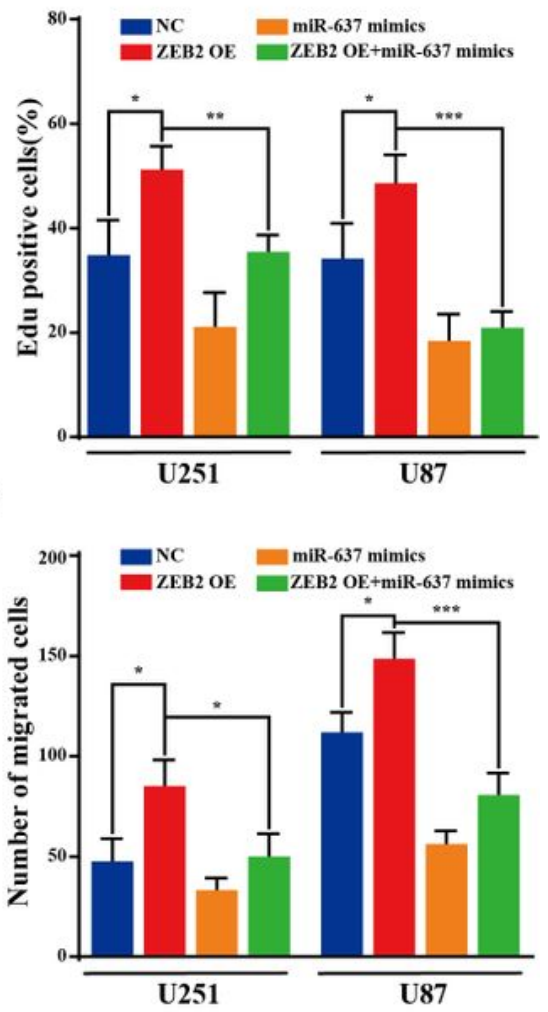

H

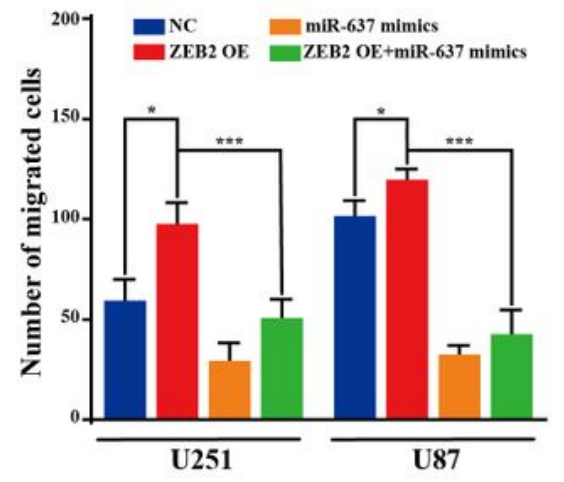

Figure 2

ZEB2 promotes the malignant phenotype of glioma by downregulating miR-637 in vitro. (A) qPCR assays demonstrated increased miR-637 levels under siZEB2 transfection. (B) The cell viability of U251 or U87 cells transfected with a ZEB2 expression plasmid and/or miR-637 lentiviral expression particles was evaluated by the MTT assay. (C-H) The proliferative rate, migration and invasion of U251 or U87 cells transfected with a ZEB2 expression plasmid and/or miR-637 mimics were evaluated by the EdU 
incorporation assay ( $C$ and $D)$, transwell assay ( $E$ and $F)$ and Boyden assay ( $G$ and $H)$, respectively. Scale bar indicates $100 \mu \mathrm{m}$. Data were presented as mean \pm S.D. $\left(* p<0.05, * \star p<0.01\right.$ and $\left.{ }^{* * *} p<0.001\right)$.
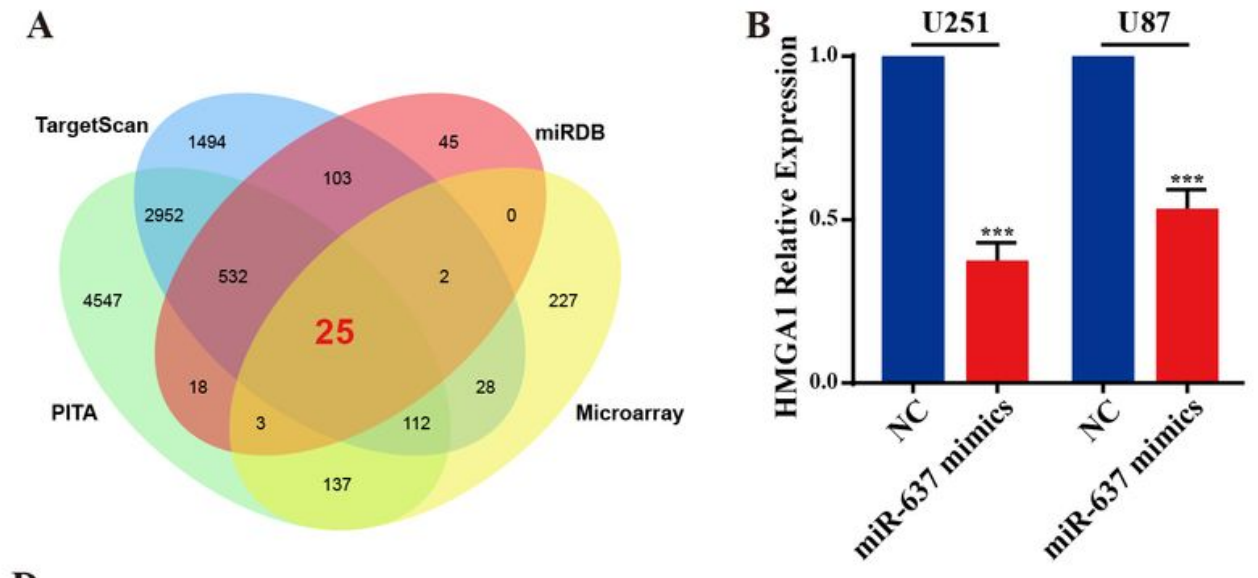

C

D

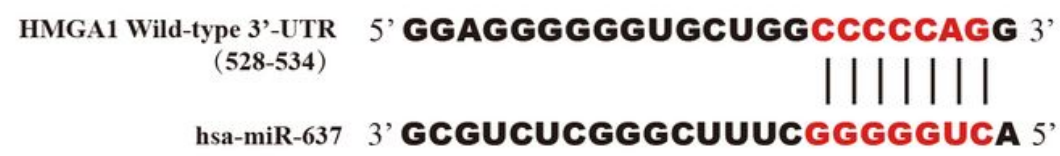

HMGA1 Mutant 3'-UTR 5' GGAGGGGGGUGCUGGAAATTGCG 3'

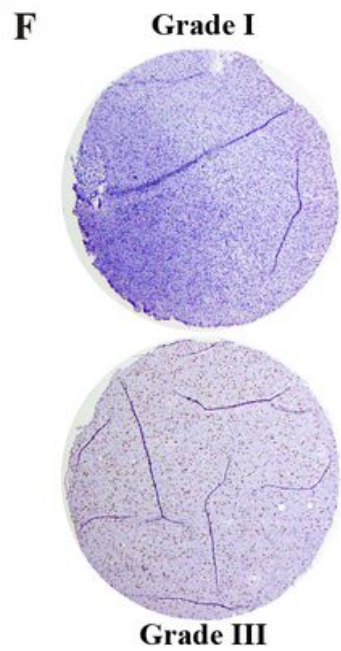

Grade III

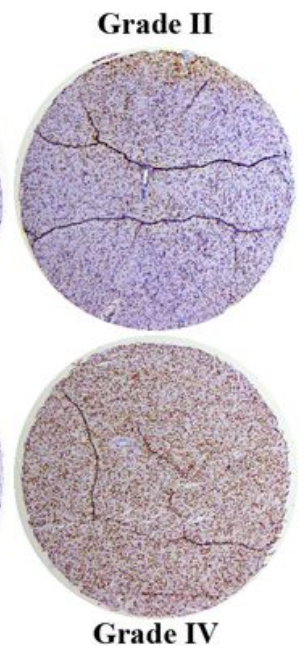

G

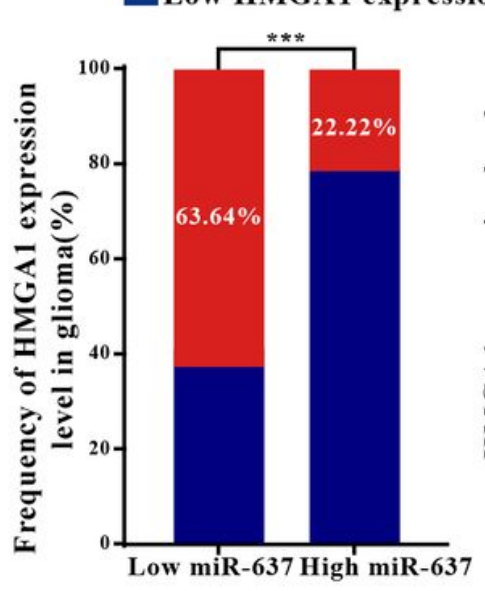

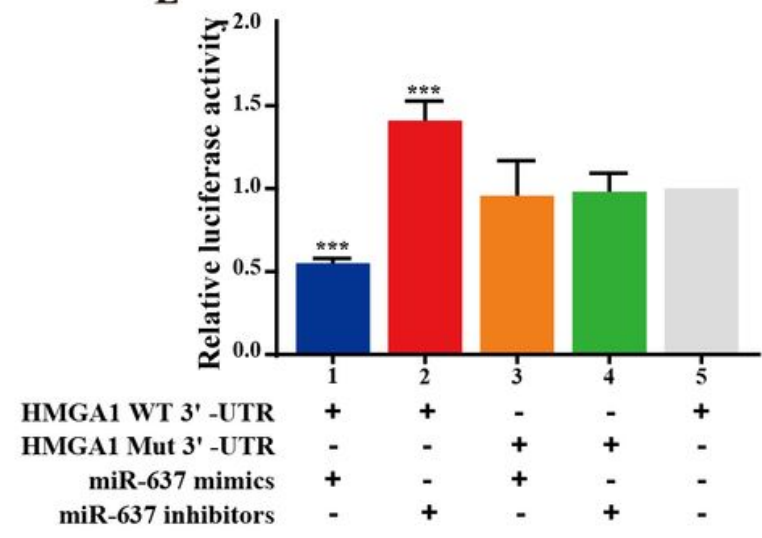

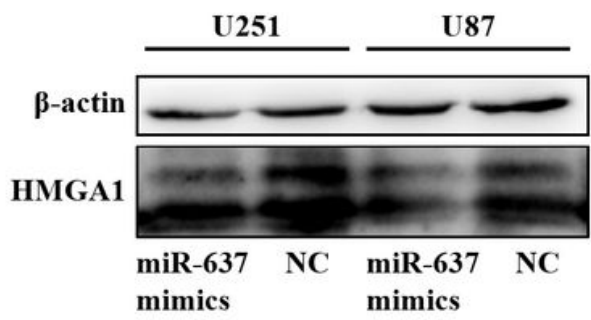

$\mathbf{E}$

\section{Figure 3}

miR-637 directly targeted HMGA1 in glioma. (A) Venn diagram indicated the intersection of miR-637 target genes predicted in public databases (TargetScan, miRDB and PICA) overlapping with microarray results. (B) qPCR assay demonstrated decreased HMGA1 levels under miR-637 mimics transfection. (C) Western blot demonstrated decreased HMGA1 levels under miR-637 mimics transfection. (D) The putative miR-637-binding sites in the 3'-UTR region of HMGA1 were marked in red. (E) Relative luciferase activity was examined after the transfection of reporter vector with HMGA1 wild-type 3囚-UTR or mutant 3囚-UTR under co-transfection with miR-637 mimics or inhibitor in 293T cells. (F) IHC analysis of HMGA1 
in glioma tissues were performed. (G) Expression of HMGA1 was negatively correlated with that of miR637 in glioma tissues. Data were presented as mean \pm S.D. $\left(* p<0.05, * \star p<0.01\right.$ and $\left.{ }^{\star * *} p<0.001\right)$. WT, wild-type; Mut, mutant.
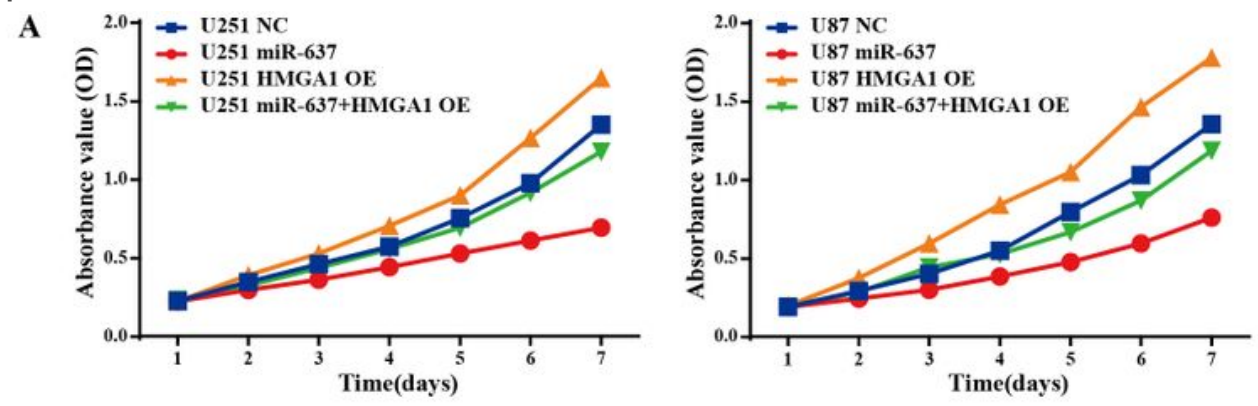

B
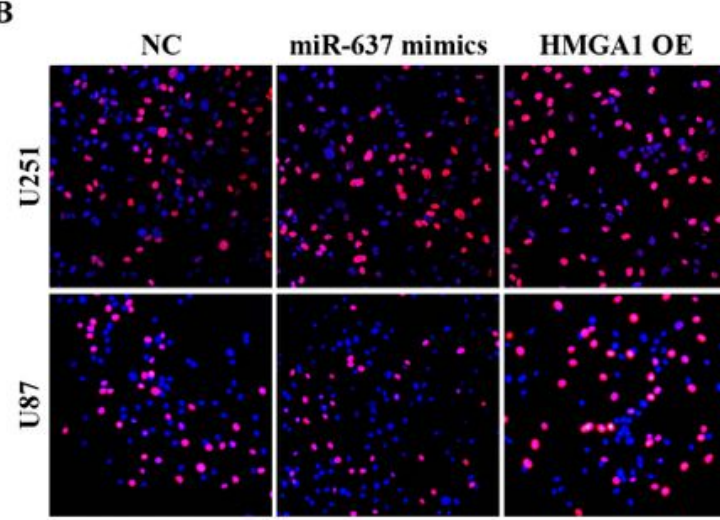

miR-637mimics +HMGA1 OE

D
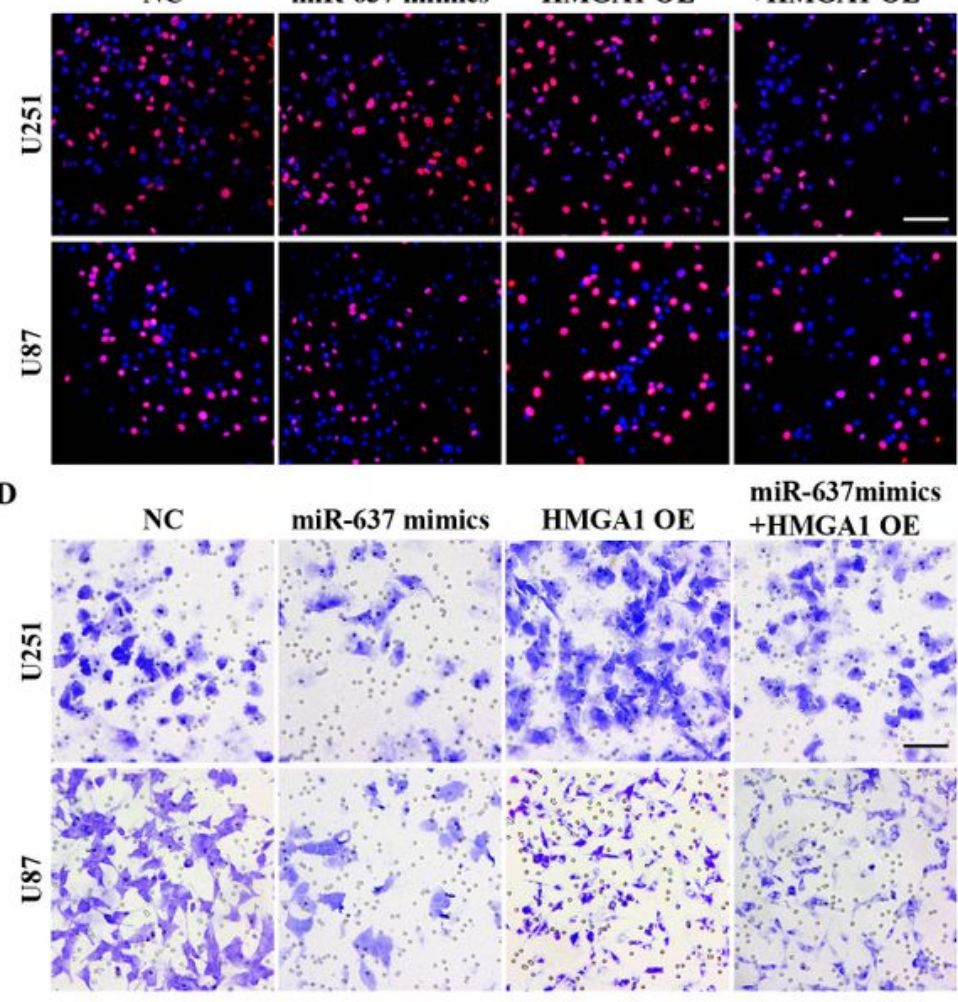

miR-637 mimics
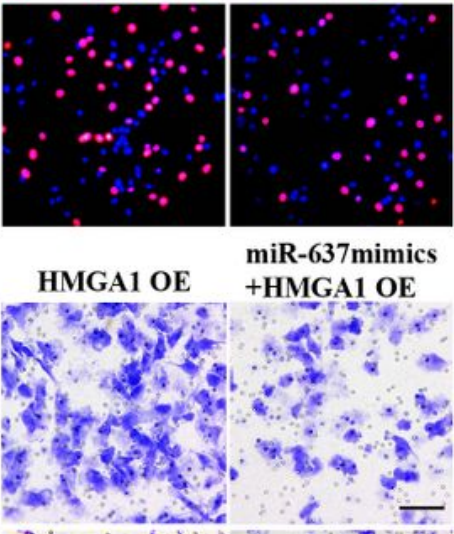

C

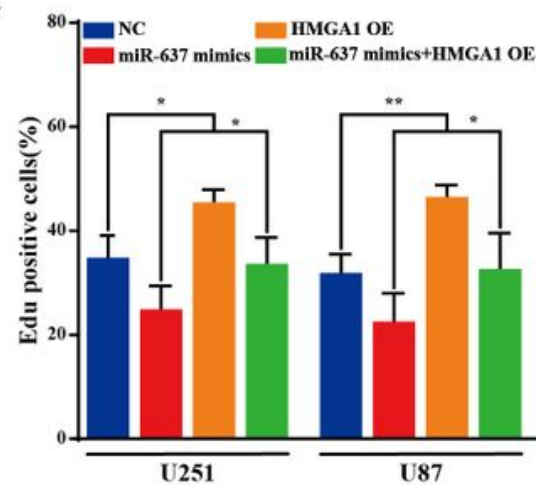

miR-637mimics

+HMGA1 OE
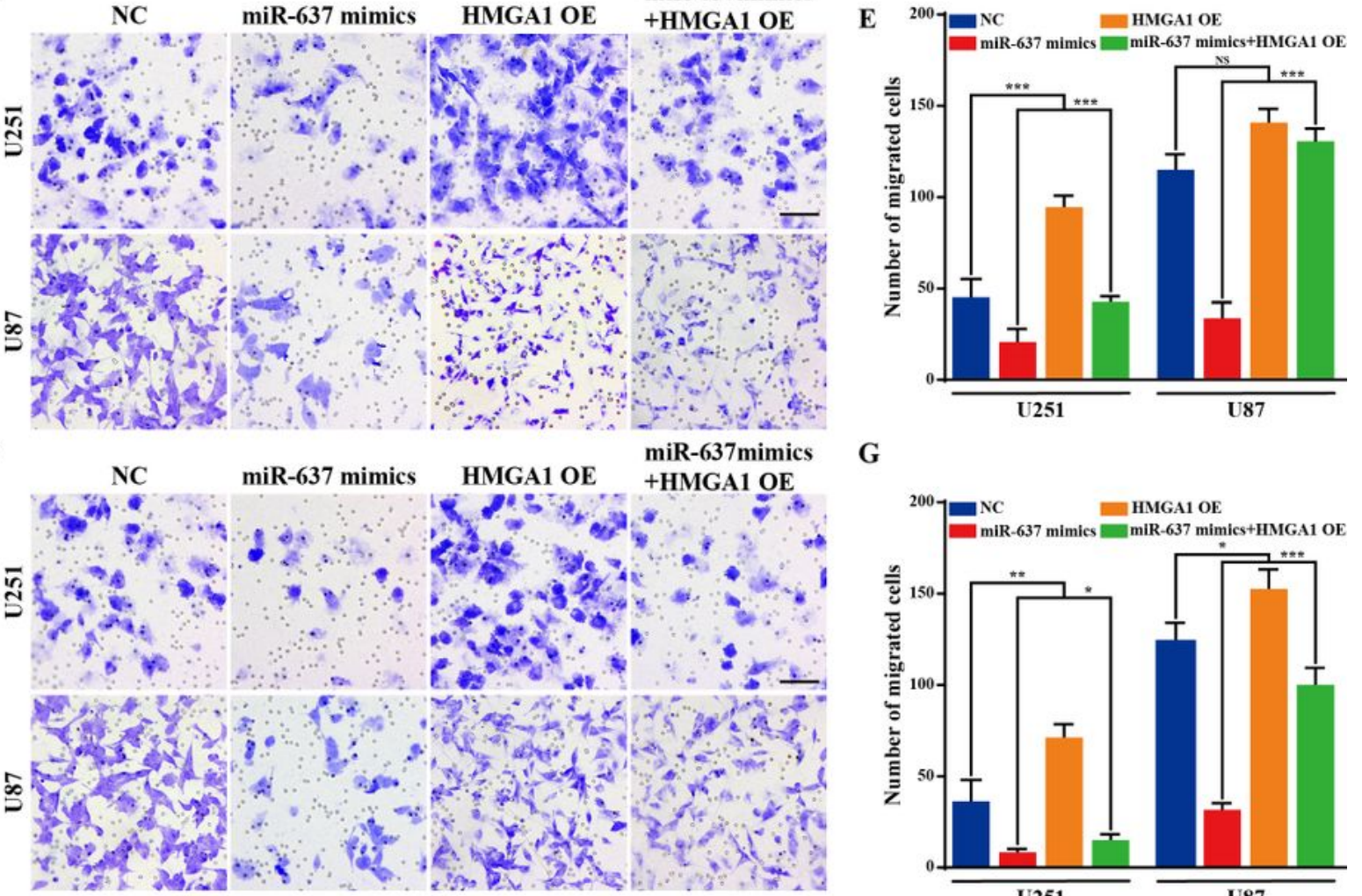

G

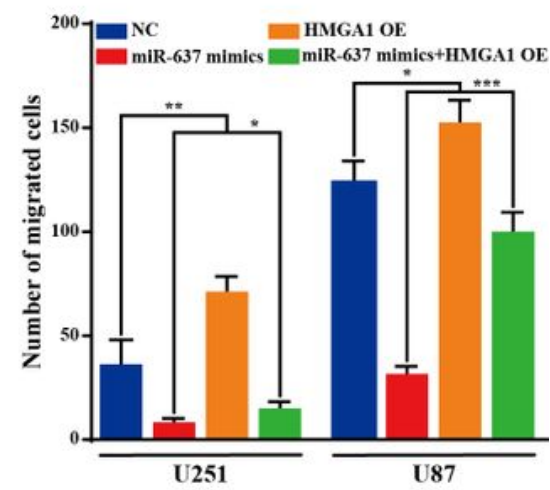

Figure 4

miR-637 suppresses the malignant phenotype of glioma by downregulating HMGA1 in vitro. (A) The cell viability of U251 or U87 cells transfected with miR-637 lentiviral expression particles and/or a HMGA1 
expression plasmid was evaluated by the MTT assay. (B-G) The proliferative rate, migration and invasion of U251 or U87 cells transfected with miR-637 mimics and/or a HMGA1 expression plasmid were evaluated by the EdU incorporation assay (B and C), transwell assay ( $D$ and $E$ ) and Boyden assay ( $F$ and $\mathrm{G})$, respectively. Scale bar indicates $100 \mu \mathrm{m}$. Data were presented as mean \pm S.D. $\left({ }^{\star} p<0.05,{ }^{\star \star} p<0.01\right.$ and $* * * p<0.001)$.

A
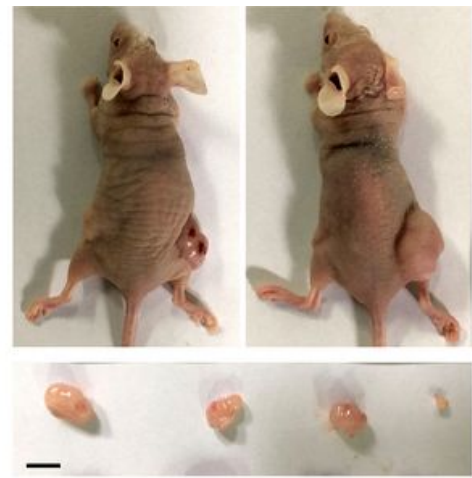

NC

B

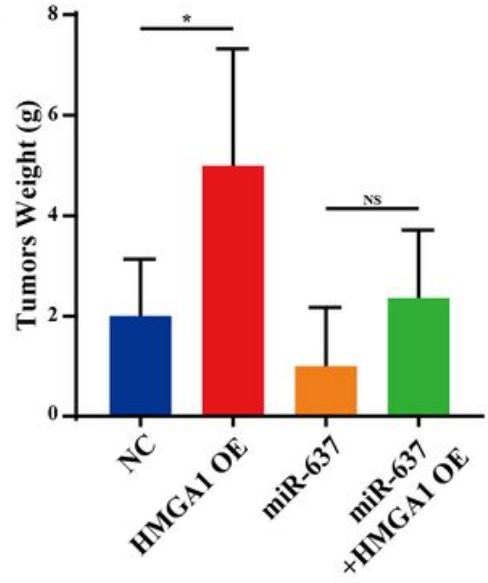

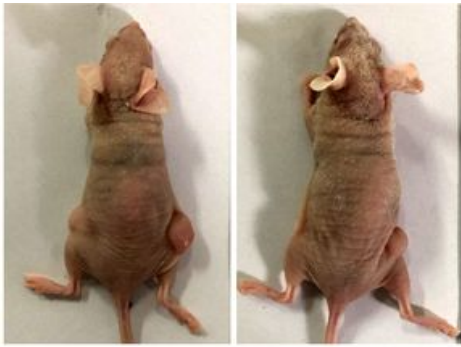

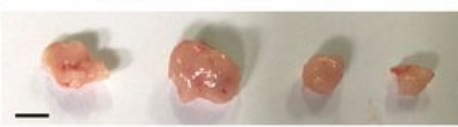

HMGA1 OE
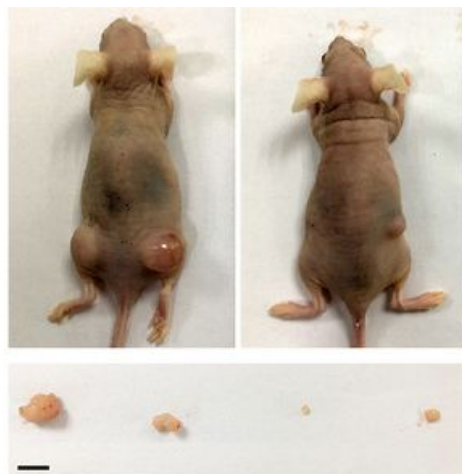

$-$

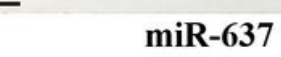

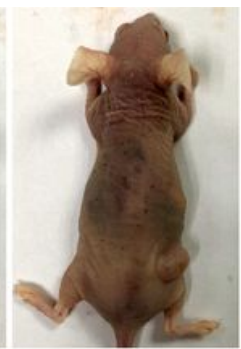

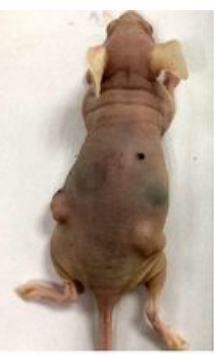

Q

miR-637+HMGA1 OE
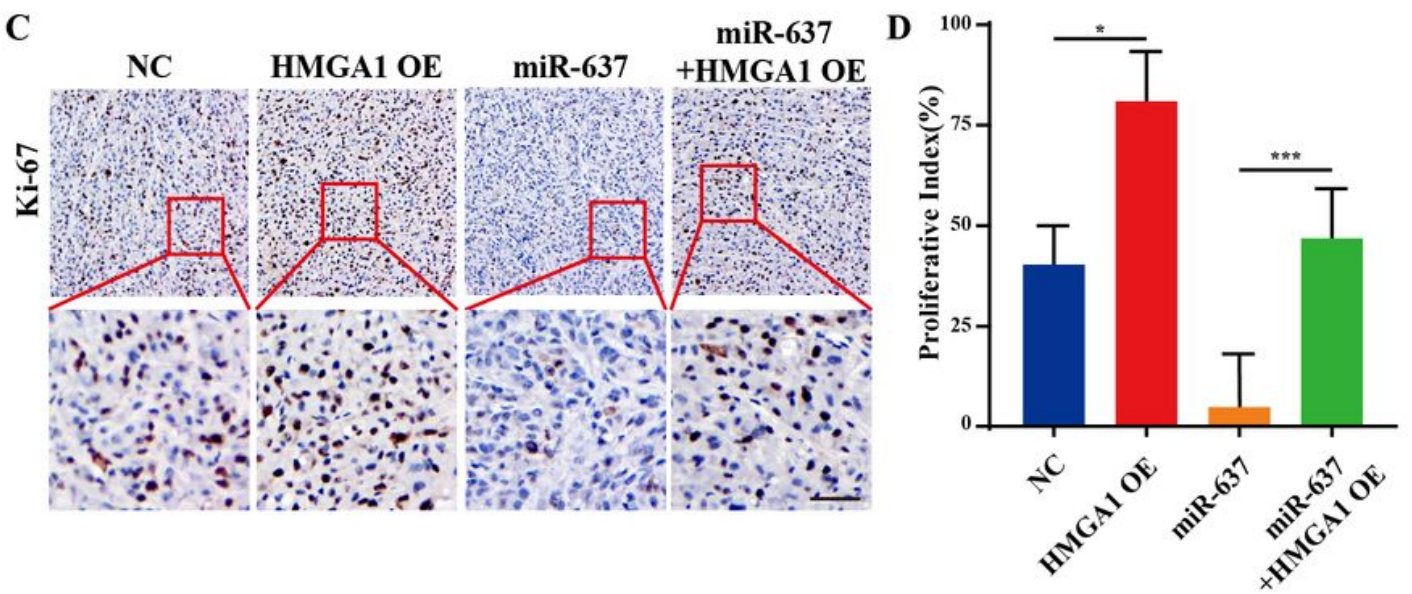

Figure 5

miR-637 modulates xenograft tumor growth by targeting HMGA1 in vivo. (A) Images of xenograft tumor models of mice injected with indicated U87 cells were shown. In the first group of four mice, U87/NC cells were injected in the left flank, and U87/HMGA1 cells were injected in the right flank; in the second group of four mice, U87/miR-637 cells were injected in the left flank, and U87/miR-637/HMGA1 cells were injected in the right flank. Scale bar indicates $1 \mathrm{~cm}$. (B) Tumor weight was measured at the 28th day after inoculation in each group. ( $C$ and $D$ ) IHC analysis and quantification of Ki-67 expression in xenograft tumors from mice injected with indicated U87 cells was performed. Scale bar indicates $100 \mu \mathrm{m}$. Data were presented as mean \pm S.D. $\left(* p<0.05, * \star p<0.01\right.$ and $\left.{ }^{*} * *<0.001\right)$. NC, negative control. 
A
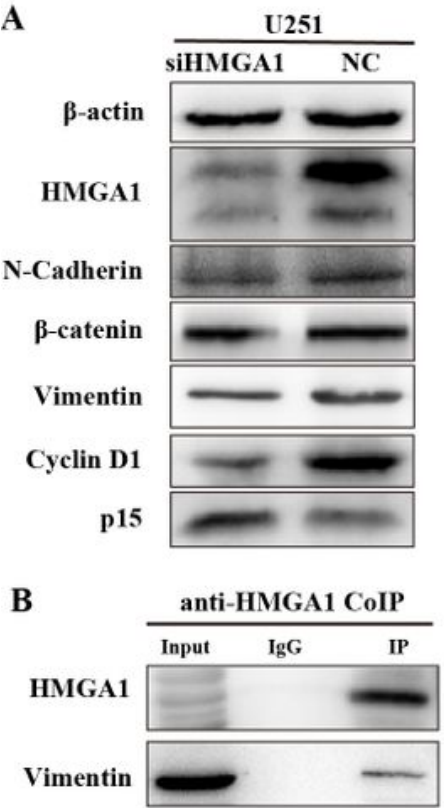

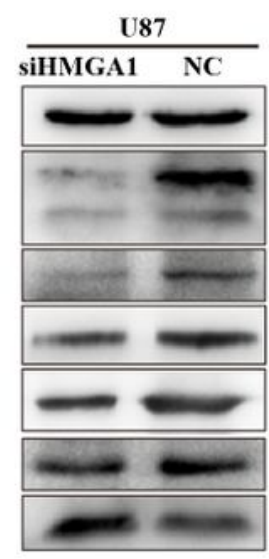

\begin{tabular}{ll}
\multicolumn{2}{c}{ anti-Vimentin CoIP } \\
\hline Input IgG IP
\end{tabular}

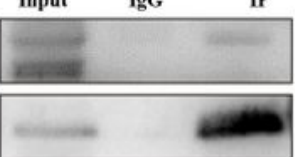

C

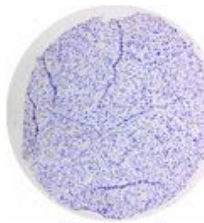

Grade I

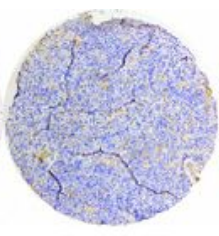

Grade II

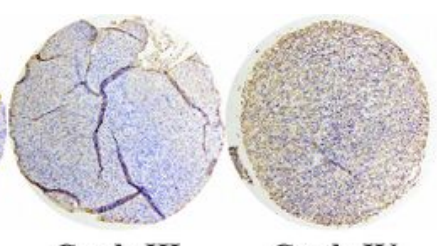

Grade III

Grade IV
D

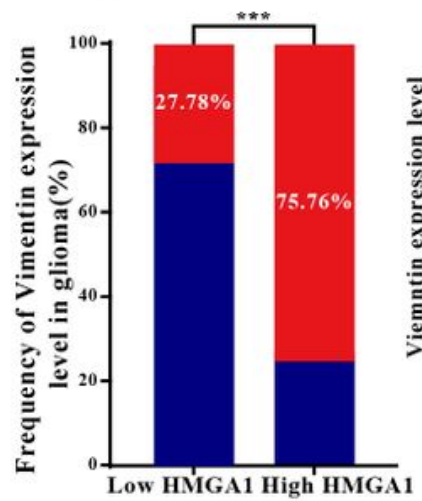

High Vimentin expression

E

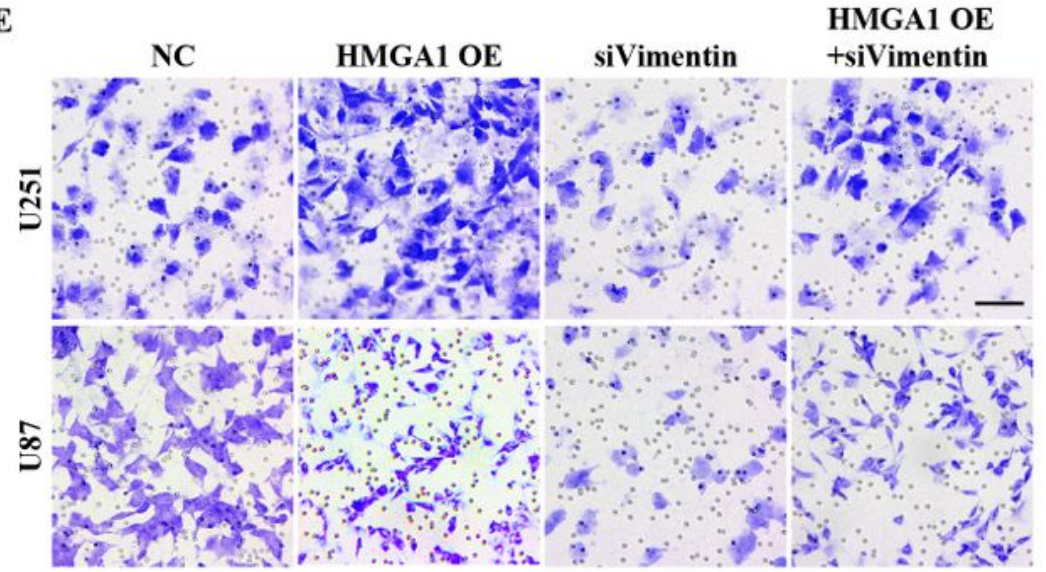

G

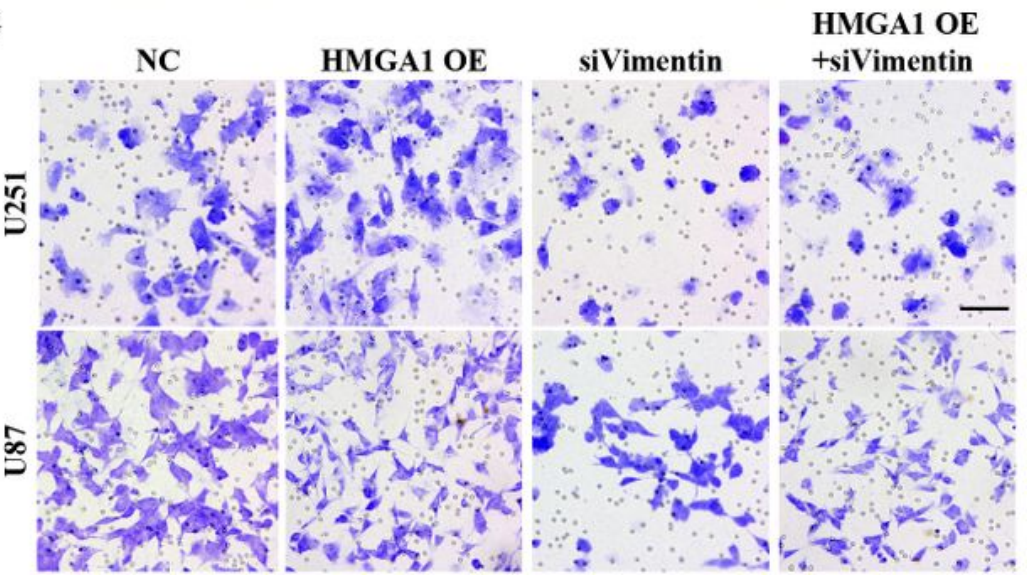

F

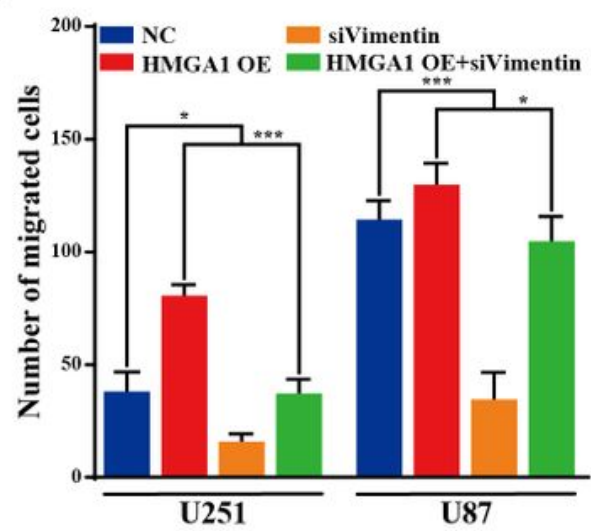

H

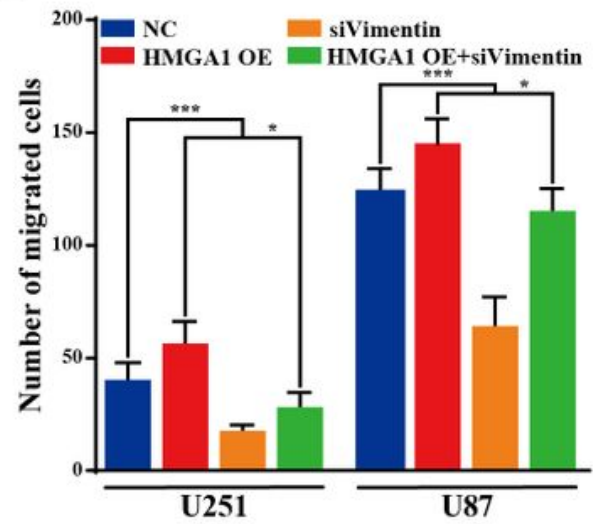

Figure 6

HMGA1 promotes glioma migration and invasion through vimentin in vitro. (A) The expression of crucial cytoskeletal-proteins and cell-cycle related proteins in indicated cells were examined by western blot assays. (B) The interaction between HMGA1 and vimentin was examined by ColP assay. (C) IHC analysis of vimentin in glioma tissues was performed. (D) Expression of Vimentin was positively correlated with that of miR-637 in glioma tissues. (E-H) Migration and invasion of U251 or U87 cells transfected with a 
HMGA1 expression plasmid and/or siRNA targeting Vimentin were evaluated by the transwell assay ( $E$ and $F$ ) and Boyden assay ( $G$ and $H)$, respectively. Scale bar indicates $100 \mu \mathrm{m}$. Data were presented as mean \pm S.D. $(* p<0.05, * \star p<0.01$ and $* \star * p<0.001)$. NC, negative control.

A

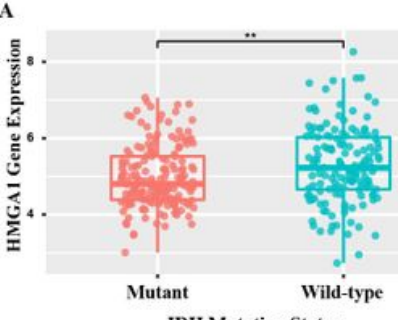

IDH Mutation Status

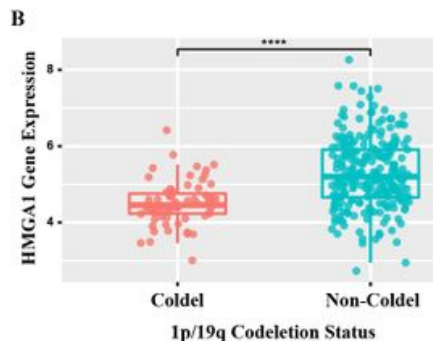

$1 \mathrm{p} / 19 \mathrm{q}$ Codeletion Status

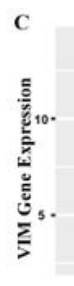

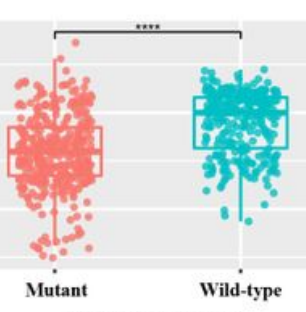

IDH Mutation Status

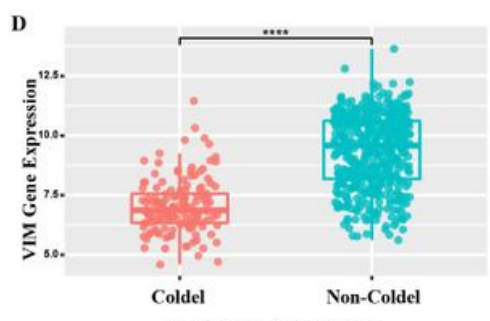

1p/19q Codeletion Status

E — High HMGA1 expression (32) — Low HMGA1 expression (33)

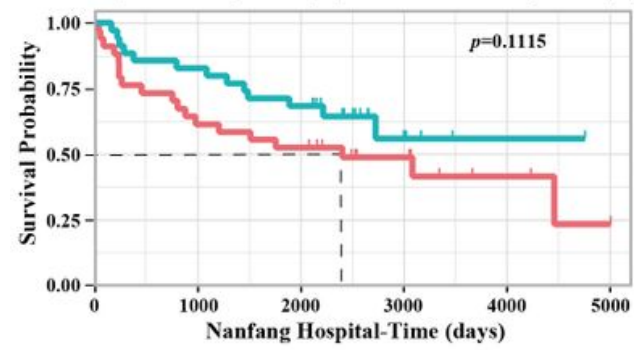

F - High HMGA1 expression (112) — Low HMGA1 expression (112)

G _ High HMGA1 expression (338) — Low HMGA1 expression (337)
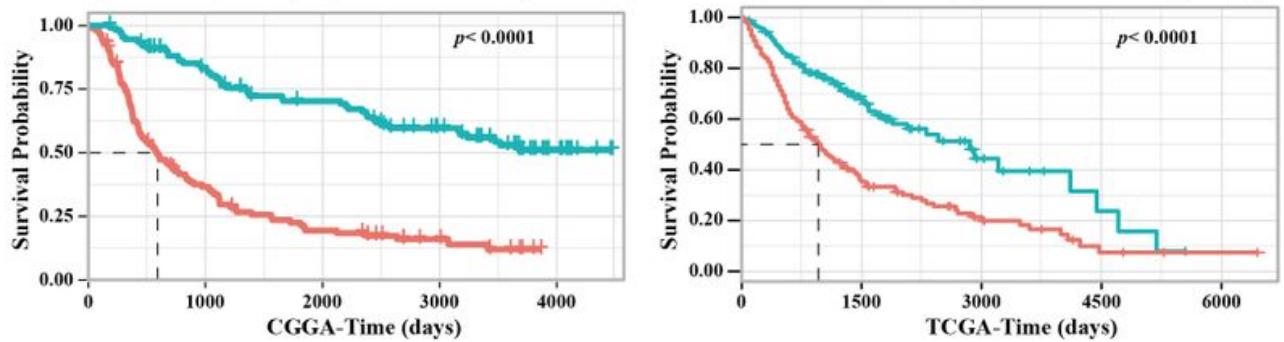

H - High Vimentin expression (32) — Low Vimentin expression (33)

$\mathbf{I}+$ High Vimentin expression (112) - Low Vimentin expression (112) $\mathbf{J}-$ High Vimentin expression (338) - Low Vimentin expression (337)
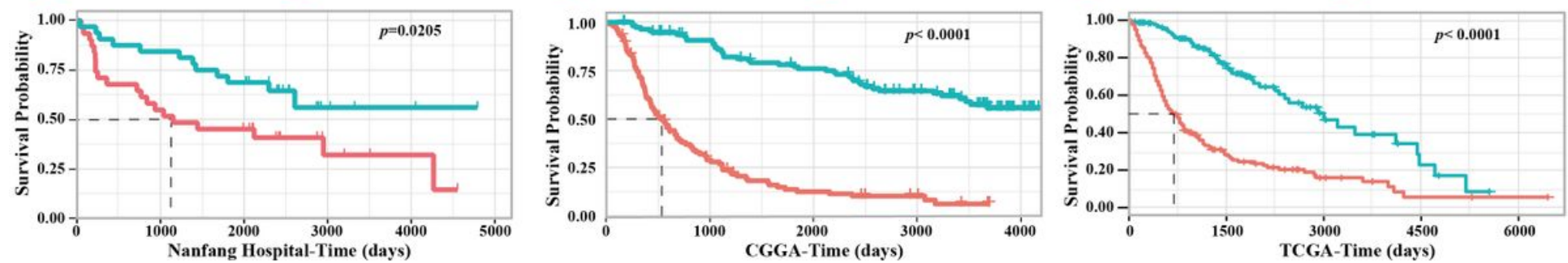

Figure 7

HMGA1 and vimentin are associated with unfavorable prognosis in glioma. (A) Expression level of HMGA1 in glioma with different IDH status in CGGA cohort was examined. (B) Expression level of HMGA1 in glioma with different 1p/19q status in CGGA cohort was examined. (C) Expression level of vimentin in glioma with different IDH status in CGGA cohort was examined. (D) Expression level of vimentin in glioma with different 1p/19q status in CGGA cohort was examined. (E-G) HMGA1 was associated with an unfavorable prognosis in Nanfang Hospital cohort, CGGA, and TCGA cohort. (H-J) Vimentin was associated with an unfavorable prognosis in Nanfang Hospital cohort, CGGA, and TCGA cohort. 


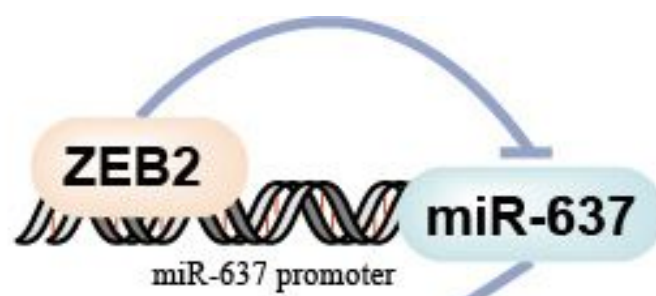

Vimentin

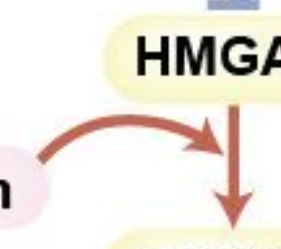

\section{HMGA1 \\ Vimentin}

\section{Migration}

\section{Invasion}

Figure 8

A schematic diagram for the oncogenic ZEB2/miR637/HMGA1 signaling axis targeting vimentin. ZEB2 could downregulate miR-637 through directly binding to the E-box element in the promoter region of miR637 , and then upregulate HMGA1, a downstream target of miR-637. This signaling axis could further increase vimentin expression and promote glioma migration and invasion.

\section{Supplementary Files}

This is a list of supplementary files associated with this preprint. Click to download.

- SupplementaryFigure1.jpg

- SupplementaryTable1.xls

- SupplementaryTable2.xls

- SupplementaryFigureLegends.doc

- SupplementaryFigure1.jpg

- SupplementaryFigure2.jpg

- SupplementaryTable1.xls

- SupplementaryTable2.xls

- SupplementaryFigure2.jpg

- SupplementaryFigureLegends.doc 